\title{
The VMC survey - XXVI. Structure of the Small Magellanic Cloud from RR Lyrae stars ${ }^{\star}$
}

\author{
T. Muraveva ${ }^{1} \dagger$, S. Subramanian ${ }^{2}$, G. Clementini ${ }^{1}$, M.-R.L. Cioni ${ }^{3,4}$, M. Palmer ${ }^{5}$, \\ J.Th. van Loon ${ }^{6}$, M. I. Moretti ${ }^{7}$, R. de Grijs ${ }^{2,8,9}$, R. Molinaro ${ }^{7}$, V. Ripepi ${ }^{7}$, \\ M. Marconi ${ }^{7}$, J. Emerson ${ }^{10}$, V. D. Ivanov ${ }^{11,12}$ \\ ${ }^{1}$ INAF-Osservatorio Astronomico di Bologna, Via Gobetti, 93/3, Bologna 40129, Italy \\ 2 Kavli Institute for Astronomy and Astrophysics, Peking University, Yi He Yuan Lu 5, Hai Dian District, Beijing 100871, China \\ 3 Leibnitz-Institut für Astrophysik Potsdam, An der Sternwarte 16, 14482 Potsdam, Germany \\ ${ }^{4}$ University of Hertfordshire, Physics Astronomy and Mathematics, College Lane, Hatfield AL10 9AB, UK \\ 5 Dept. d'Astronomia i Meteorologia, Institut de Ciències del Cosmos, Universitat de Barcelona (IEEC-UB), Martí Franquès 1, \\ E-08028 Barcelona, Spain \\ 6 Lennard-Jones Laboratories, Keele University, ST5 5BG, UK \\ 7 INAF-Osservatorio Astronomico di Capodimonte, via Moiariello 16, Naples 80131, Italy \\ 8 Department of Astronomy, Peking University, Yi He Yuan Lu 5, Hai Dian District, Beijing 100871, China \\ ${ }^{9}$ International Space Science Institute-Beijing, 1 Nanertiao, Zhongguancun, Hai Dian District, Beijing 100190, China \\ 10 Astronomy Unit, School of Physics and Astronomy, Queen Mary University of London, Mile End Road, London E1 4NS, UK \\ 11 European Southern Observatory, Ave. Alonso de Córdova 3107, Vitacura, Santiago, Chile \\ 12 European Southern Observatory, Karl-Schwarzschild-Strasse 2, D-85748 Garching bei München, Germany
}

Accepted . Received ; in original form

\begin{abstract}
We present results from the analysis of 2997 fundamental mode RR Lyrae variables located in the Small Magellanic Cloud (SMC). For these objects near-infrared time-series photometry from the VISTA survey of the Magellanic Clouds system (VMC) and visual light curves from the OGLE IV survey are available. In this study the multi-epoch $K_{\mathrm{s}}$-band VMC photometry was used for the first time to derive intensity-averaged magnitudes of the SMC RR Lyrae stars. We determined individual distances to the RR Lyrae stars from the near-infrared period-absolute magnitude-metallicity $\left(P M_{K_{\mathrm{s}}} Z\right)$ relation, which has some advantages in comparison with the visual absolute magnitudemetallicity $\left(M_{V}-[\mathrm{Fe} / \mathrm{H}]\right)$ relation, such as a smaller dependence of the luminosity on interstellar extinction, evolutionary effects and metallicity. The distances we have obtained were used to study the three-dimensional structure of the SMC. The distribution of the SMC RR Lyrae stars is found to be ellipsoidal. The actual line-of-sight depth of the SMC is in the range from 1 to $10 \mathrm{kpc}$, with an average depth of $4.3 \pm 1.0 \mathrm{kpc}$. We found that RR Lyrae stars in the eastern part of the SMC are affected by interactions of the Magellanic Clouds. However, we do not see a clear bimodality observed for red clump (RC) stars, in the distribution of RR Lyrae stars.
\end{abstract}

Key words: Surveys - stars: variables: RR Lyrae - galaxies: Magellanic Clouds galaxies: structure

\section{INTRODUCTION}

The Small Magellanic Cloud (SMC) is a nearby dwarf irregular galaxy. It is part of the Magellanic System (MS) which also comprises the Large Magellanic Cloud (LMC), the Magellanic Bridge (MB) and the Magellanic Stream. The SMC gravitationally interacts with the LMC and the Milky Way

\footnotetext{
* Based on observations made with VISTA at ESO under programme ID 179.B-2003.

† tatiana.muraveva@oabo.inaf.it
}

(MW). As a result it has a complex internal structure characterised by a disturbed shape and a large extent along the line-of-sight (Putman et al. 1998).

RR Lyrae stars are old (age $>10$ Gyr), low-mass $\left(\sim 0.6-0.8 M_{\odot}\right)$, radially-pulsating variables located on the horizontal branch of the colour-magnitude diagram (CMD). They pulsate in the fundamental mode (RRab), first-overtone mode (RRc) or both modes simultaneously (RRd). RR Lyrae stars are abundant in globular clusters and in the halos of galaxies. They could serve to study the structure, interaction history and the distance to the 
parent systems because they follow an absolute magnitudemetallicity $\left(M_{V}-[\mathrm{Fe} / \mathrm{H}]\right)$ relation in the visual band and infrared period-luminosity $(P L)$ and $P L$-metallicity $(P L Z)$ relations.

The three-dimensional structure of the SMC as traced by RR Lyrae stars has been the subject of a number of studies (e.g. Graham 1975; Soszyński et al. 2010; Subramanian \& Subramaniam 2012; Haschke et al. 2012; Kapakos \& Hatzidimitriou 2012; Deb et al. 2015; Jacyszyn-Dobrzeniecka et al. 2016, Deb 2017). Graham (1975) analysed 76 SMC RR Lyrae stars finding that these objects are distributed smoothly and do not show a strong concentration in the bar or in the centre of the SMC. Soszyński et al. (2010) published the catalogue of RR Lyrae stars observed in the SMC by the third phase of the Optical Gravitational Lensing Experiment (OGLE III) and concluded that the distribution of RR Lyrae stars in the galaxy is roughly round on the sky with two maxima near the centre. Subramanian \& Subramaniam (2012) analysed the relative positions of different regions of the SMC inferred from the $V$ and $I$ photometry of the RR Lyrae variables observed by OGLE III. According to this study the SMC RR Lyrae stars have an ellipsoidal distribution and the northeastern part of the SMC is located closer to us. Similarly, Haschke et al. (2012) used the SMC RR Lyrae stars from the OGLE III survey and found that the RR Lyrae stars show a spheroidal or ellipsoidal distribution with an off-centered and nearly bimodal peak. Deb et al. (2015) found that the north-eastern arm of the SMC is located closer than the plane of the SMC main body. These authors studied the depth along the line-of-sight and concluded that it is larger for the central part of the SMC. Kapakos \& Hatzidimitriou (2012) studied a sample of RRab stars located in $14 \mathrm{deg}^{2}$ of the SMC and found that the north-eastern part of the SMC has a greater depth. Furthermore, they studied the metal abundance and the spatial distribution of the RR Lyrae variables and suggested that the metal-richer and metal-poorer objects in the sample belong to different dynamical structures. Recently, Jacyszyn-Dobrzeniecka et al. (2016) studied the RR Lyrae stars using OGLE IV data, which covers almost entirely the SMC. They confirm the ellipsoidal shape of the SMC and do not find any sub-structures. However, they detect some asymmetry in the equal density contours in the eastern part of the SMC. Namely, the center of the ellipsoid shifts to east and towards the observer. Deb (2017) used the sample of the OGLE IV RRab stats to determine the distance, reddening and structural parameters of the SMC.

In all studies mentioned above visual photometry was used to analyse the SMC RR Lyrae stars. In the present study, for the first time, $K_{\mathrm{s}}$-band multi-epoch photometry from the VISTA survey of the Magellanic Clouds system (VMC) for 2997 RRab stars distributed across a large area $\left(\sim 42 \mathrm{deg}^{2}\right)$ of the SMC, was used to probe the galaxy's structure. The near-infrared ( $K$ or $K_{\mathrm{s}}$ bands) period-absolute magnitude-metallicity $\left(P M_{K} Z\right)$ relation has some advantages in comparison with the optical $M_{V}-[\mathrm{Fe} / \mathrm{H}]$ relation, specifically, a smaller dependence of the luminosity on interstellar extinction $\left(A_{K}=0.114 A_{V}\right)$, evolutionary effects and metallicity. It was discovered by Longmore et al. (1986) and later studied by different authors from both a theoretical and an observational point of view (e.g. Bono et al. 2003; Catelan et al. 2004; Di Criscienzo, Marconi \& Caputo
2004; Del Principe et al. 2006; Sollima et al. 2006, 2008; Borissova et al. 2009; Marconi et al. 2015; Muraveva et al. 2015). Here we apply the relation from Muraveva et al. (2015) to derive individual distances to RR Lyrae stars in the SMC sample and study the structure of this galaxy.

In Section 2 we provide information about our sample of RR Lyrae variables in the SMC and the data available for these objects from the VMC and OGLE IV surveys. In Section 3 we analyse the $P K_{\mathrm{s}}$ relations for the whole sample of SMC RR Lyrae stars as well as for individual VMC tiles. We present the three-dimensional structure of the SMC as traced by RR Lyrae stars in Section 4. Finally, Section 5 provides a summary of our results and main conclusions.

\section{DATA}

\subsection{The VMC survey}

VMC (Cioni et al. 2011) is an ongoing imaging survey of the MS in the $Y, J, K_{\mathrm{s}}$ passbands, centred at $\lambda=1.02,1.25$ and $2.15 \mu \mathrm{m}$, respectively. It started in 2009 and observations of the MS were $89 \%$ complete as of September 2017, while the observations of the SMC were $100 \%$ complete. The survey covers the LMC area $\left(\sim 105 \mathrm{deg}^{2}\right)$ with 68 tiles, the SMC $\left(\sim 42 \mathrm{deg}^{2}\right)$ with 27 tiles, the MB area $\left(\sim 21 \mathrm{deg}^{2}\right)$ with 13 tiles and part of the Stream $\left(\sim 3 \mathrm{deg}^{2}\right)$ with 2 tiles. The VMC $K_{\mathrm{s}}$-band observations are taken over 13 separate epochs: 11 times with an exposure time of $750 \mathrm{~s}$ (deep epochs) and twice with an exposure time of $375 \mathrm{~s}$ (shallow epochs). Additional epochs may be obtained if observations have to be repeated because the requested sky conditions are not met (Cioni et al. 2011). Every single deep epoch reaches a limiting magnitude of $K_{\mathrm{s}} \sim 19.2 \mathrm{mag}$ with a signal-to-noise ratio $S / N=5$, in the Vega system. VMC reaches a sensitivity limit on the stacked images of $K_{\mathrm{s}}=21.5 \mathrm{mag}$ with $S / N=5$. The VMC images are processed by the Cambridge Astronomical Survey Unit (CASU; Lewis, Irwin \& Bunclark 2010). The data are then sent to the Wide Field Astronomy Unit (WFAU) in Edinburgh where the single epochs are stacked, catalogued and ingested into the VISTA Science Archive (VSA; Cross et al. 2012).

The strategy, main science goals and the first data from the VMC survey were described in Cioni et al. (2011). Analysis of variable stars based on VMC data was presented in Ripepi et al. (2012a,b), Ripepi et al. (2016), Moretti et al. (2016) and Marconi et al. (2017) for classical Cepheids, Ripepi et al. (2014) for Anomalous Cepheids, Ripepi et al. (2015) for Type II Cepheids, in Moretti et al. (2014) for classical Cepheids, RR Lyrae stars and eclipsing binaries (EBs), in Muraveva et al. (2015) for RR Lyrae stars and in Muraveva et al. (2014) for EBs. Using the VMC observations of the red clump (RC) stars Subramanian et al. (2017) and Tatton et al. (2013) studied the structure of the SMC and 30 Doradus region in the LMC, respectively. Rubele et al. (2015) analysed the star formation history of the SMC using the VMC photometry.

To study the $K_{\mathrm{s}}$-band light curves of the SMC RR Lyrae stars we used VMC observations in all 27 SMC tiles. We analysed the $K_{\mathrm{s}}$-band light curves with the GRaphical Analyser of TImes Series (GRATIS), custom software developed at the Observatory of Bologna by P. Montegriffo 
Table 1. VMC tiles in the SMC analysed in the present study: (1) Field and tile number; (2), (3) Coordinates of the tile centre J2000; (4) Number of epochs in the $K_{\mathrm{S}}$ band, available at the moment of analysis including observations obtained in nights with sky conditions that did not meet the VMC requirements (see text for details) (5) Number of RRab stars; (6) PK $K_{\mathrm{s}}$ relation of RR Lyrae stars in the tile; (7) r.m.s. of the relation; (8) Distance moduli of the tiles.

\begin{tabular}{|c|c|c|c|c|c|c|c|}
\hline Tile & $\begin{array}{c}\alpha \\
\left(\mathrm{h}:{ }^{\mathrm{m}}: \mathrm{s}\right)\end{array}$ & $\begin{array}{c}\delta \\
\left({ }^{\circ}:^{\prime}:^{\prime \prime}\right)\end{array}$ & $\begin{array}{c}\mathrm{N} \\
\text { epochs }\end{array}$ & $\begin{array}{c}\mathrm{N}_{\mathrm{RR} \text { Lyr }} \\
(\%)\end{array}$ & $\begin{array}{c}P K_{\mathrm{s}} \\
\text { relation }\end{array}$ & $\begin{array}{l}\text { r.m.s. } \\
\text { (mag) }\end{array}$ & $\begin{array}{c}(m-M)_{0} \\
\quad(\mathrm{mag})\end{array}$ \\
\hline SMC 2_2 & $00: 21: 43.920$ & $-75: 12: 04.320$ & 12 & 67 & $(-3.03 \pm 0.46) \log P+(17.71 \pm 0.10)$ & 0.15 & $18.87 \pm 0.18$ \\
\hline SMC 2_3 & 00:44:35.904 & $-75: 18: 13.320$ & 15 & 103 & $(-3.02 \pm 0.46) \log P+(17.71 \pm 0.10)$ & 0.16 & $18.89 \pm 0.18$ \\
\hline SMC 2_4 & 01:07:33.864 & $-75: 15: 59.760$ & 15 & 93 & $(-2.89 \pm 0.39) \log P+(17.72 \pm 0.09)$ & 0.14 & $18.86 \pm 0.15$ \\
\hline SMC 2_5 & $01: 30: 12.624$ & $-75: 05: 27.600$ & 16 & 76 & $(-2.01 \pm 0.60) \log P+(17.89 \pm 0.14)$ & 0.19 & $18.84 \pm 0.20$ \\
\hline SMC 3_1 & 00:02:39.912 & $-73: 53: 31.920$ & 16 & 26 & $(-4.14 \pm 0.54) \log P+(17.43 \pm 0.12)$ & 0.13 & $18.82 \pm 0.17$ \\
\hline SMC 3_2 & $00: 23: 35.544$ & $-74: 06: 57.240$ & 14 & 102 & $(-2.96 \pm 0.36) \log P+(17.75 \pm 0.08)$ & 0.14 & $18.88 \pm 0.19$ \\
\hline SMC 3_3 & 00:44:55.896 & $-74: 12: 42.120$ & 18 & 207 & $(-3.41 \pm 0.28) \log P+(17.64 \pm 0.07)$ & 0.16 & $18.91 \pm 0.18$ \\
\hline SMC 3_4 & 01:06:21.120 & $-74: 10: 38.640$ & 16 & 190 & $(-3.45 \pm 0.27) \log P+(17.60 \pm 0.06)$ & 0.16 & $18.86 \pm 0.17$ \\
\hline SMC 3_5 & $01: 27: 30.816$ & $-74: 00: 49.320$ & 16 & 96 & $(-2.33 \pm 0.41) \log P+(17.83 \pm 0.09)$ & 0.17 & $18.85 \pm 0.19$ \\
\hline SMC 3_6 & 01:48:06.120 & $-73: 43: 28.200$ & 16 & 38 & $(-2.28 \pm 0.80) \log P+(17.77 \pm 0.18)$ & 0.19 & $18.78 \pm 0.20$ \\
\hline SMC 4_1 & 00:05:33.864 & $-72: 49: 12.000$ & 13 & 41 & $(-2.05 \pm 0.64) \log P+(17.95 \pm 0.14)$ & 0.14 & $18.85 \pm 0.26$ \\
\hline SMC $4 \_2$ & $00: 25: 14.088$ & $-73: 01: 47.640$ & 15 & 142 & $(-3.22 \pm 0.29) \log P+(17.72 \pm 0.07)$ & 0.13 & $18.94 \pm 0.19$ \\
\hline SMC $4 \_3$ & 00:45:14.688 & $-73: 07: 11.280$ & 16 & 279 & $(-3.57 \pm 0.31) \log P+(17.61 \pm 0.07)$ & 0.24 & $18.90 \pm 0.27$ \\
\hline SMC $4 \_4$ & 01:05:19.272 & $-73: 05: 15.360$ & 15 & 250 & $(-4.12 \pm 0.24) \log P+(17.48 \pm 0.06)$ & 0.17 & $18.88 \pm 0.21$ \\
\hline SMC $4 \_5$ & 01:25:11.088 & $-72: 56: 02.760$ & 18 & 124 & $(-2.20 \pm 0.50) \log P+(17.86 \pm 0.11)$ & 0.19 & $18.84 \pm 0.21$ \\
\hline SMC 4_6 & 01:44:34.512 & $-72: 39: 44.640$ & 16 & 42 & $(-3.13 \pm 0.82) \log P+(17.64 \pm 0.19)$ & 0.18 & $18.85 \pm 0.18$ \\
\hline SMC $5 \_2$ & $00: 26: 41.688$ & $-71: 56: 35.880$ & 17 & 89 & $(-3.01 \pm 0.45) \log P+(17.74 \pm 0.10)$ & 0.14 & $18.88 \pm 0.19$ \\
\hline SMC 5_3 & $00: 45: 32.232$ & $-72: 01: 40.080$ & 19 & 174 & $(-3.32 \pm 0.29) \log P+(17.67 \pm 0.06)$ & 0.15 & $18.91 \pm 0.16$ \\
\hline SMC 5_4 & 01:04:26.112 & $-71: 59: 51.000$ & 18 & 188 & $(-3.02 \pm 0.34) \log P+(17.72 \pm 0.08)$ & 0.20 & $18.89 \pm 0.23$ \\
\hline SMC 5_5 & 01:23:09.336 & $-71: 51: 09.720$ & 14 & 111 & $(-3.57 \pm 0.45) \log P+(17.56 \pm 0.10)$ & 0.17 & $18.82 \pm 0.21$ \\
\hline SMC 5_6 & 01:41:28.800 & $-71: 35: 47.040$ & 18 & 53 & $(-3.30 \pm 0.58) \log P+(17.60 \pm 0.13)$ & 0.14 & $18.83 \pm 0.14$ \\
\hline SMC 6_2 & 00:28:00.192 & $-70: 51: 21.960$ & 17 & 83 & $(-1.57 \pm 0.45) \log P+(18.05 \pm 0.10)$ & 0.14 & $18.90 \pm 0.16$ \\
\hline SMC 6_3 & $00: 45: 48.792$ & $-70: 56: 09.240$ & 14 & 120 & $(-3.01 \pm 0.30) \log P+(17.72 \pm 0.07)$ & 0.14 & $18.87 \pm 0.17$ \\
\hline SMC 6_4 & 01:03:40.152 & $-70: 54: 25.200$ & 13 & 100 & $(-3.00 \pm 0.31) \log P+(17.72 \pm 0.07)$ & 0.14 & $18.87 \pm 0.17$ \\
\hline SMC 6_5 & $01: 21: 22.560$ & $-70: 46: 11.640$ & 14 & 71 & $(-3.06 \pm 0.46) \log P+(17.70 \pm 0.10)$ & 0.14 & $18.86 \pm 0.19$ \\
\hline SMC 7_3 & 00:46:04.728 & $-69: 50: 38.040$ & 16 & 56 & $(-2.70 \pm 0.56) \log P+(17.81 \pm 0.13)$ & 0.14 & $18.91 \pm 0.16$ \\
\hline SMC 7_4 & 01:03:00.480 & $-69: 48: 58.320$ & 15 & 76 & $(-3.37 \pm 0.47) \log P+(17.63 \pm 0.11)$ & 0.14 & $18.88 \pm 0.15$ \\
\hline
\end{tabular}

(see e.g. Clementini et al. 2000). This software requires at least 11 epochs to model the light curves and properly compute intensity-averaged magnitudes. However, even though all sources located in selected tiles are expected to have 13 good quality epochs, stars affected by some problems, such as blending, may not necessarily be detected in all epochs and, thus, have the 11 data points necessary for the analysis with GRATIS. Hence, initially we used all available VMC epochs including observations obtained in nights with sky conditions (seeing and ellipticity) that did not meet the VMC requirements (Cioni et al. 2011) and discarded data points that deviated significantly from the model fit during the analysis with GRATIS. For some stars the model line fits well all data points, hence, we used all available data in the analysis. The VMC coverage of the SMC is shown in Figure 1, where red boxes represent the 27 SMC tiles $\left(\sim 42 \operatorname{deg}^{2}\right)$ used in this study. The $X$ and $Y$ axes in Fig. 1 are the coordinates of a zenithal equidistant projection as defined by van der Marel \& Cioni (2001).

The time sampling of the VMC survey and the significantly reduced amplitudes of the light variations in the $K_{\mathrm{s}}$ passband allow us to determine mean $K_{\mathrm{s}}$ magnitudes for RR Lyrae and other pulsating stars with great precision (Ripepi et al. 2012a, 2014). On the other hand, the small amplitude of pulsation in the $K_{\mathrm{s}}$ band complicates the search for new variables based solely on near-infrared data. A method to identify variable stars in the MS based only on the VMC photometry was developed by Moretti et al. (2016), but it is more suited to search for classical Cepheids. Hence, our study is based only on known SMC variable stars identified by optical microlensing surveys such as OGLE IV (Soszyński et al. 2016).

\subsection{OGLE IV}

The initial goal of the OGLE survey was the search for microlensing events, but as a byproduct the survey also discovered a large number of variable stars in the MS and in the MW bulge. The OGLE IV catalogue comprises observations of about $650 \mathrm{deg}^{2}$ in the MS obtained between March 2010 and July 2015 with the $1.3 \mathrm{~m}$ Warsaw telescope at the Las Campanas Observatory, Chile (Udalski, Szymański \& Szymański 2015). Observations were performed in the Cousins $I$ passband with the number of data points ranging from 100 to 750 and in the Johnson $V$ band with the number of observations ranging from several to 260 . The OGLE IV catalogue is publicly available from the OGLE website ${ }^{1}$ and contains, among others, $45451 \mathrm{RR}$ Lyrae variables, of which 6369 stars are located towards the SMC (Soszyński et al. 2016). The catalogue provides right ascension (RA), declination (Dec), mode of pulsation, mean $V$ and $I$ magnitudes, period of pulsation, $I$-band amplitude,

1 http://ogle.astrouw.edu.pl 


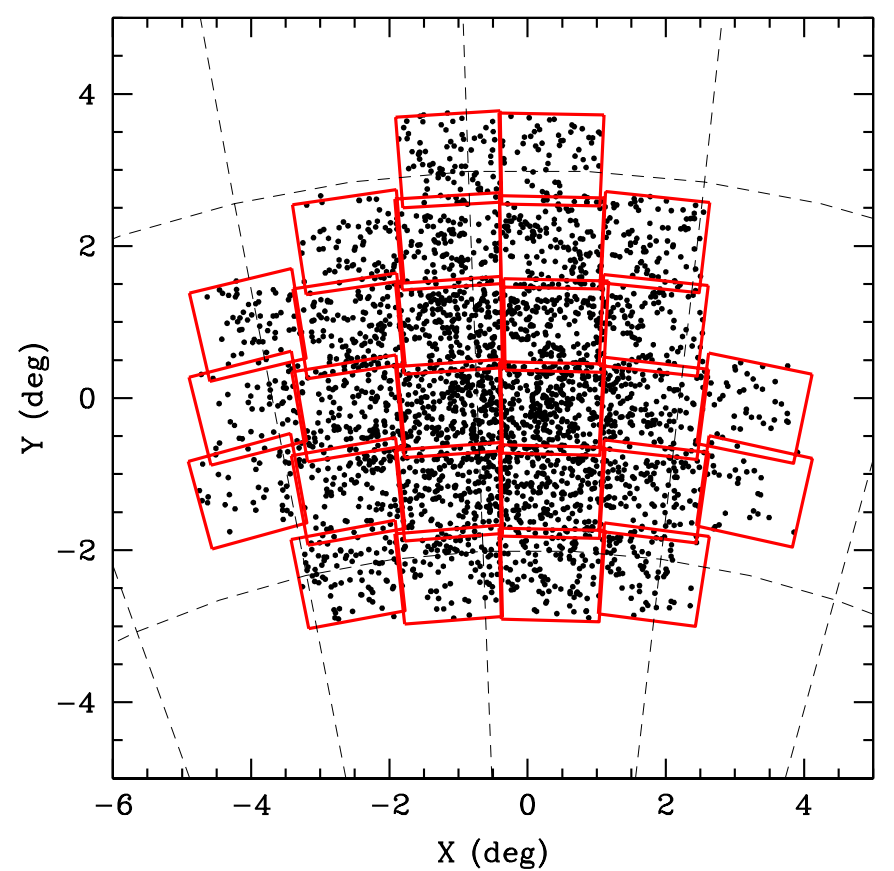

Figure 1. VMC coverage of the SMC. Red boxes represent 27 VMC tiles. Black dots mark the 2997 RR Lyrae stars analysed in the this paper. Coordinates are defined as in van der Marel \& Cioni (2001) where $\alpha_{0}=12.5 \mathrm{deg}, \delta_{0}=-73$ deg.

parameters of the Fourier decomposition and time-series $V, I$ photometry.

Among the SMC RR Lyrae variables we selected only RRab stars for our analysis, since RRc and RRd stars have smaller amplitudes and noisier light curves, hence, it is particularly complicated to fit their near-infrared light curves and obtain reliable mean $K_{\mathrm{s}}$ magnitudes. OGLE IV provides information on 4961 RRab stars in the SMC, of which 3484 variables have counterparts in the VMC catalogue within $1^{\prime \prime}$. Among 1477 variables that do not have VMC counterpart within $1^{\prime \prime}$, 1336 are located outside the VMC footprint, while 141 stars are in the VMC footprint but we did not find them likely because of coordinates' uncertainty. Adopting a cross-matching radius of $2 \operatorname{arcsec}$ instead of 1 arcsec we were able to recover VMC counterparts for 100 among 141 stars, and 125 stars are recovered within a radius of 5 arcsec. However, it is risky to add these stars to the sample, since wrong crossmatches could happen. Stars that do not have a VMC counterpart even within 5 arcsec are aligned along the edges of the VMC footprint, located in the gap between VMC tiles 5_3 and 5_4 or are clustered in some kind of holes of the VMC coverage likely caused by saturated MW stars in front of the SMC. However, the percentage of objects that we lose owing to these issues is rather small.

We discarded from the sample of 3484 variables which have counterparts in the VMC catalogue within $1^{\prime \prime}$, stars that have fewer than $11 K_{\mathrm{S}}$ band epochs (see Section 2.1) and sources observed by VISTA detector 16 . Detector 16 is affected by a time-varying quantum efficiency which makes accurate flat fielding impossible (Jarvis et al. 2013). This effect is more significant in the shorter wavelength filters but also present in the $K_{\mathrm{s}}$ band. After these cleaning procedures we are left with a sample of $3121 \mathrm{RR}$ Lyrae variables.
We analysed the $K_{\mathrm{s}}$-band light curves of the $3121 \mathrm{RR}$ Lyrae stars with GRATIS, and derived intensity-averaged magnitudes and amplitudes using the periods provided by OGLE IV. Examples of the $K_{\mathrm{s}}$-band light curves are shown in Fig. 2, where black and green dots represent the data points used and discarded from the analysis, respectively. Red lines represent the best fits obtained with GRATIS.

The distribution of the $3121 \mathrm{RR}$ Lyrae stars in the $K_{\mathrm{s}}$ magnitude versus logarithmic period plane is shown in Fig. 3. The large majority of the stars follow the $P K_{\mathrm{s}}$ relation. However, there is a group of objects that do not follow the $P K_{\mathrm{s}}$ relation and have significantly brighter magnitudes. They are likely MW members or sources blended with close companions. A division between SMC and MW RR Lyrae stars is not provided in the OGLE IV catalogue since Soszyński et al. (2016) pointed out that it was impossible to separate the MW and SMC old stellar populations owing to the interaction between the two galaxies. However, in the current analysis we want to focus on the SMC inner structure. Hence, we perform an approximate separation between the two populations and clean the sample from blended sources. In order to do this we perform a linear nonweighted least squares fit to the whole sample of $3121 \mathrm{RR}$ Lyrae variables (red line in Fig. 3) by progressively discarding objects which deviate more than $5 \sigma$ from the best fit line (122 sources in total, shown by green squares in Fig. 3 ). These RR Lyrae stars are likely MW variables or blended sources.

We chose a rather large scatter $\left(5 \sigma\right.$ from the $P K_{\mathrm{s}}$ relation) to distinguish objects that belong to the SMC because the actual dispersion of the $P K_{\mathrm{s}}$ relation in Fig. 3 includes not only the intrinsic dispersion of the relation, but also the scatter caused by the different distances spanned by the RR Lyrae stars in our sample (depth effect). Selecting stars located within a smaller interval, for example $3 \sigma$, is risky, since it causes the removal of RR Lyrae variables that actually belong to the SMC but are significantly scattered from the $P K_{\mathrm{s}}$ owing to the large extension of the SMC along the line-of-sight.

Of the 122 stars that scatter more than $5 \sigma$ from the $P K_{\mathrm{s}}$ relation 72 are too bright also in the OGLE IV catalogue and significantly scattered from the $P L$ relation in $I$ band, hence, they are either MW members or stars blended in both, the $I$ and $K_{\mathrm{s}}$ passbands. We discarded them from the following analysis. Additional 50 objects have $V$ and $I$ magnitudes consistent with the distance to the SMC, but too bright magnitudes in the $K_{\mathrm{s}}$ passband. We checked their OGLE and VMC images and found that all of them have close companions and are blended in the $K_{\mathrm{s}}$ band, hence should be discarded. We are thus left with a sample of 2999 RR Lyrae stars. Classification of one of them (OGLE-SMCRRLYR-1505) was marked as uncertain in the OGLE IV catalogue. Another object (OGLE-SMC-RRLYR-3630) represents two RRab stars with different periods located on the line of sight. We analysed the $K_{\mathrm{s}}$-band light curve of this star using both periods but we were not able to find out which one of the two periods is correct owing to a small number of data points in the $K_{\mathrm{s}}$ band. Thus, we discarded stars OGLE-SMC-RRLYR-1505 and OGLE-SMC-RRLYR3630 from the analysis, hence, our final sample comprises 2997 RR Lyrae variables, which are shown as black dots in Fig. 1 and their main properties are presented in Table 3. 

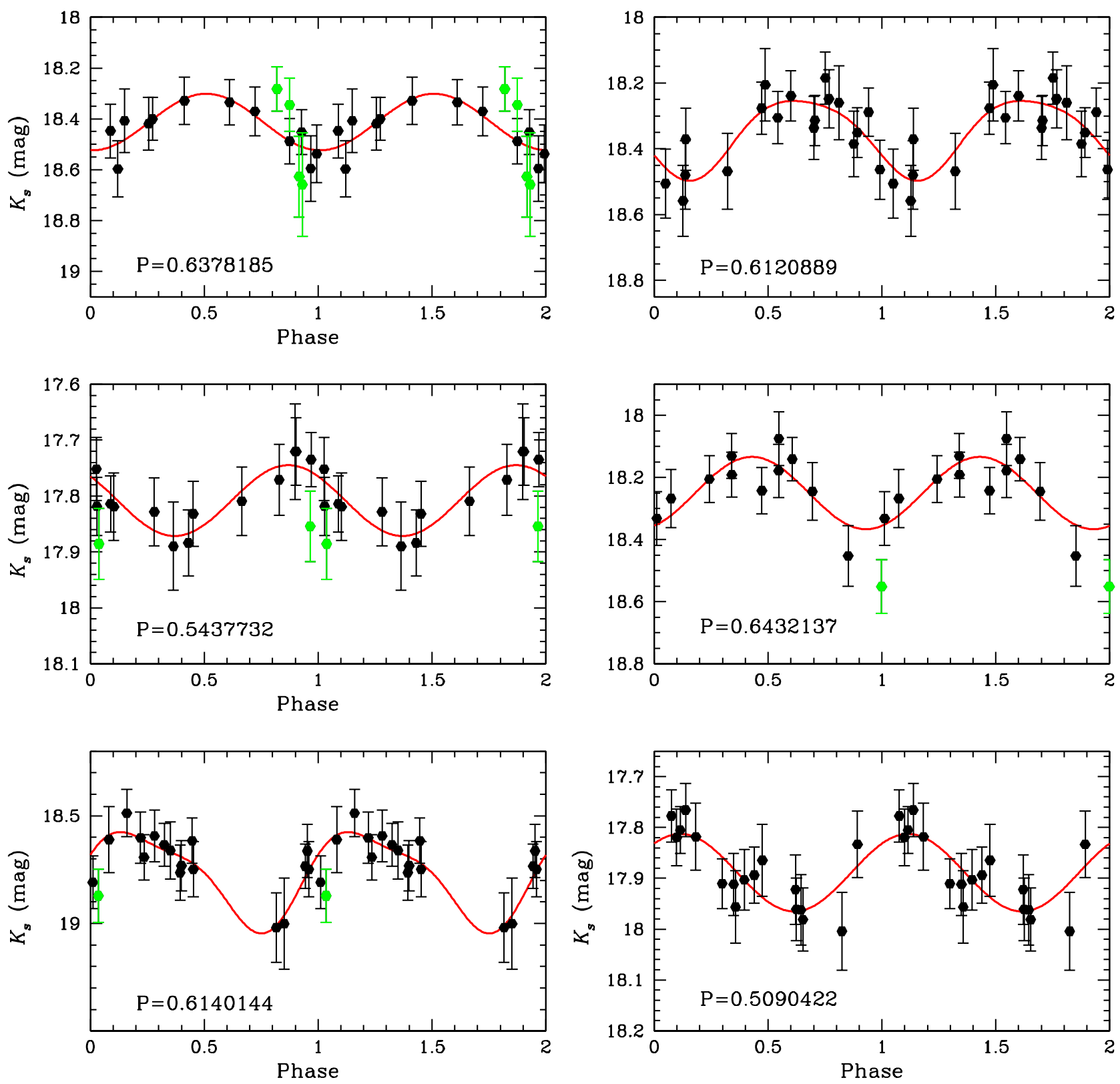

Figure 2. Phased $K_{\mathrm{s}}$-band light curves of the SMC RRab stars observed by the VMC survey with 11 or more epochs. Periods are from the OGLE IV catalogue and measured in days. Black and green dots represent data points used and discarded from the analysis, respectively. The red line shows the best fit model obtained with GRATIS.

\subsection{Extinction}

The reddening in the SMC has been studied by several authors (e.g. Schlegel et al. 1998; Zaritsky et al. 2002; Israel et al. 2010; Haschke et al. 2011; Subramanian \& Subramaniam 2012, Deb 2017). In this study we have obtained our own estimation of the reddening for the $2997 \mathrm{RR}$ Lyrae variables in our sample from the difference between their intrinsic and observed colours:

$E(V-I)=(V-I)-(V-I)_{0}$
Piersimoni, Bono \& Ripepi (2002) developed an empirical relation that connects the intrinsic colour $(V-I)_{0}$ of RRab stars to the $V$-band amplitude and period:

$(V-I)_{0}=(0.65 \pm 0.02)-(0.07 \pm 0.01) A m p(V)+(0.36 \pm 0.06) \log P$

The OGLE IV catalogue provides amplitudes in the $I$ passband. We transformed them to $V$-band amplitudes using the relation developed as a byproduct during the work for the Gaia first data release (DR1, Clementini et al. 2016). The relation was derived using a large sample of RR Lyrae 


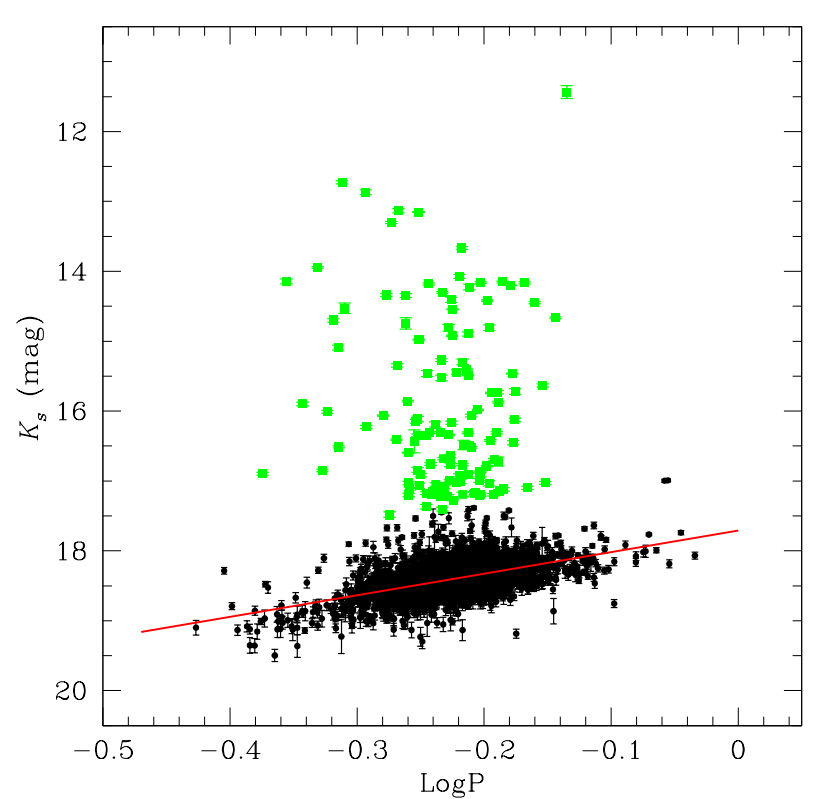

Figure 3. $P K_{\mathrm{S}}$ relation of $3121 \mathrm{SMC}$ RRab stars from the OGLE IV catalogue which have a counterpart and 11 or more $K_{\mathrm{s}}$-band epochs in VMC and were not observed by VISTA detector 16. The red line represents the best non-weighted least squares fit. Black circles and green squares are stars located within and beyond $5 \sigma$ from the best fit line, respectively.

stars which also includes SMC's RR Lyrae variables (Ripepi et al. in preparation).

$$
A m p(V)=(1.487 \pm 0.013) A m p(I)+(0.042 \pm 0.007)
$$

The r.m.s. of the relation is $0.03 \mathrm{mag}$. We calculated the intrinsic colours of the 2997 RR Lyrae variables in our sample using Eqs. 2-3. The uncertainties in $(V-I)_{0}$ were calculated by error propagation adopting the r.m.s. of Eq. 3 as an uncertainty in $A m p(V)$. Then we calculated individual reddening values for all 2997 variables using Eq. 1 and $V$ and $I$ apparent magnitudes from OGLE IV. Uncertainties in apparent $V$ and $I$ magnitudes are not provided in the OGLE IV catalogue. Following Jacyszyn-Dobrzeniecka et al. (2016) we assumed their values as 0.02 mag. Reddening values and related uncertainties obtained by this procedure are provided in Table 3.

For 27 RRab stars in the sample it was not possible to estimate the reddening since their $V$ apparent magnitudes are not available in the OGLE IV catalogue. The mean reddening of the remaining $2970 \mathrm{RR}$ Lyrae variables is $\langle E(V-I)\rangle=0.06 \pm 0.06 \mathrm{mag}$. We assigned this mean value to the 27 stars for which a direct determination of reddening was not possible.

Reddening estimates bear uncertainties which may lead to negative reddening values. For 232 stars out of 2997 (8\% of the sample) we found negative reddening values. However, 165 of them have reddening consistent with zero within the uncertainties, which means that only 67 variables $(2 \%$ of the sample) have really negative values. The lowest reddening in the sample is $E(V-I)=-0.56 \mathrm{mag}$, while the median reddening of all stars with negative values is $E(V-I)=$ $-0.02 \mathrm{mag}$. If negative reddening values are ignored, this will skew the distribution towards positive reddening values and

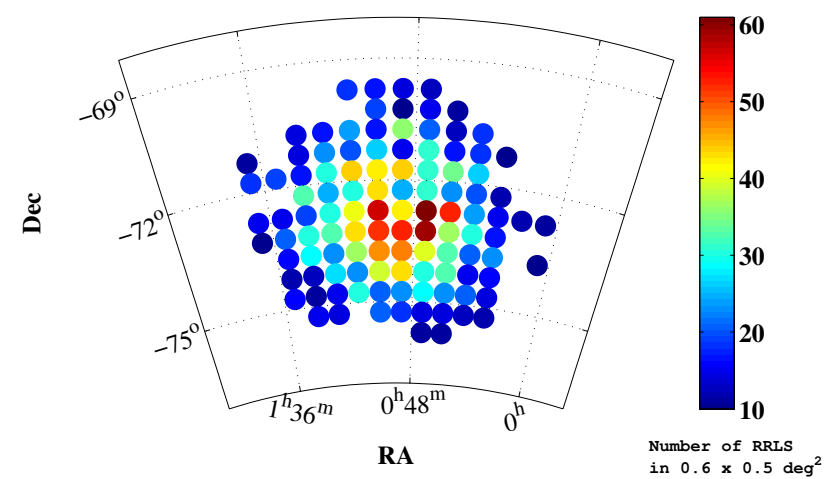

Figure 4. Two-dimensional distribution of the number of stars in different sub-regions of the SMC. Each point corresponds to a sub-region of $0.6 \times 0.5 \mathrm{deg}^{2}$ area, for a total number of 110 sub-regions.

leads to bias. By retaining negative reddening values, the uncertainties will be propagated properly. Hence, we keep the negative values as a reflection of the uncertainties in reddening in the following analysis.

We have divided the SMC region in the small subregions of equal area $\left(0.6 \times 0.5 \mathrm{deg}^{2}\right)$. Only those subregions which have at least $10 \mathrm{RR}$ Lyrae variables $(3 \times$ Poissonian error) are considered for the analysis. There are 110 sub-regions which satisfy this criterion. The number of stars in sub-regions range from 10 to 60 . More specifically, the number of stars is $10-30$ in the outer regions and $30-60$ in the inner regions of the SMC. Fig. 4 shows the distribution of stars in each of 110 sub-regions. In Fig. 5 we show the mean extinction values of RR Lyrae variables located in the sub-regions. The extinction is larger in the eastern/south-eastern parts of the SMC. Obtained extinction maps are very similar to the extinction maps produced by Subramanian \& Subramaniam (2012) and Haschke et al. (2011) using RC stars, and Deb (2017) using RR Lyrae stars. We discuss this in details in Section 4.1.

In order to calculate dereddened $K_{\mathrm{s}, 0}$ magnitudes we applied the relations $E(V-I)=1.22 E(B-V)$ and $A_{K}=$ $0.114 A_{V}$ (Cardelli et al. 1989, Caputo, Marconi \& Musella 2000) thus obtaining:

$K_{\mathrm{s}, 0}=K_{\mathrm{s}}-0.29 E(V-I)$

The mean extinction $A_{K_{\mathrm{s}}}$ of the 2997 RR Lyrae stars in our sample is $0.02 \mathrm{mag}$, which is significantly smaller than the typical uncertainty of the $K_{\mathrm{s}}$ individual mean apparent magnitudes (0.07 mag). Schlegel et al. (1998) estimated the typical reddening towards the SMC from the median dust emission in surrounding annuli and found the value $E(B-V)=0.037 \mathrm{mag}$ which corresponds to $E(V-I)=$ $0.045 \mathrm{mag}$. This value is smaller than the value of reddening estimated in this paper $E(V-I)=0.06 \pm 0.06 \mathrm{mag}$, but is in agreement with it within the errors. Zaritsky et al. (2002) 


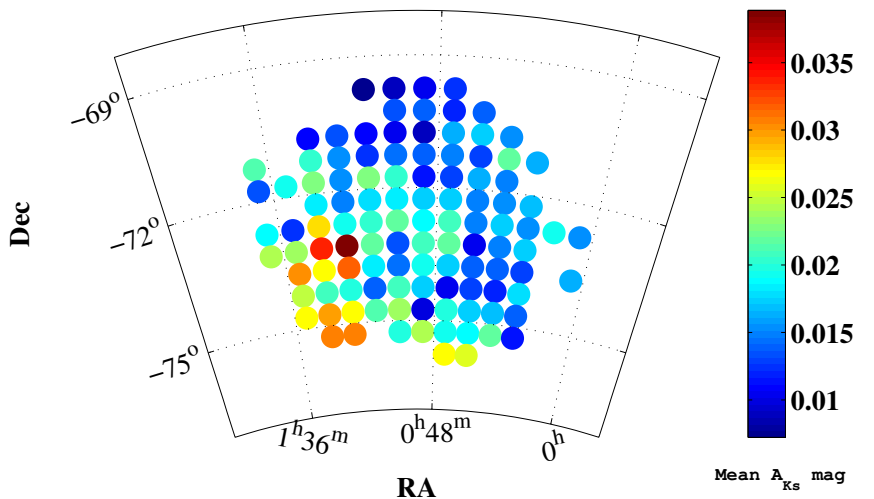

Figure 5. Two-dimensional distribution of the mean extinction measured in different sub-regions of the SMC. Each point corresponds to a sub-region of $0.6 \times 0.5 \mathrm{deg}^{2}$ area, for a total number of 110 sub-regions.

produced an extinction map across the SMC and studied the nature of extinction as a function of stellar population. Particularly, the authors derived the extinction values for the cooler older stars $\left(5500 \mathrm{~K} \leq T_{\text {eff }} \leq 6500 \mathrm{~K}\right)$ and for the hotter younger stars $\left(12000 \mathrm{~K} \leq T_{\text {eff }} \leq 45000 \mathrm{~K}\right)$. Zaritsky et al. (2002) found that the mean extinction is lower for the cooler population $\left(A_{V}=0.18 \mathrm{mag}\right)$. This corresponds to $A_{K}=0.02 \mathrm{mag}$, which is equal to the mean extinction value that we found for 2997 RR Lyrae stars in the SMC. Israel et al. (2010) estimated the mean internal extinction of the SMC $A_{V}=0.45 \mathrm{mag}$, which corresponds to $A_{K}=0.05 \mathrm{mag}$ and is higher than the mean extinction $A_{K}=0.02 \mathrm{mag}$ found in this paper for RR Lyrae stars. Subramanian \& Subramaniam (2012) estimated reddening of the SMC using RC stars and found the mean reddening towards the SMC $E(V-I)=0.053 \pm 0.017$ mag. Haschke et al. (2011) found mean reddening of the SMC $E(V-I)=0.04 \pm 0.06$ mag using RC stars and a mean value of $E(V-I)=0.07 \pm 0.06$ mag from RR Lyrae stars. Deb (2017) estimated mean reddening value of the SMC using OGLE IV RR Lyrae stars as $E(B-V)=0.056 \pm 0.019$ mag, which corresponds to $E(V-I)=0.068$ mag. All estimates of the reddening in the SMC based on $\mathrm{RC}$ and $\mathrm{RR}$ Lyrae stars are consistent with the value found in this study.

The individual dereddened $K_{\mathrm{s}, 0}$ magnitudes of $2997 \mathrm{RR}$ Lyrae stars in the SMC are listed in column 7 of Table 3 and used in the following analysis to derive the individual distance to each RR Lyrae star in the sample.

\section{PERIOD-LUMINOSITY RELATION}

We performed a non-weighted linear least squares fit of the $P K_{\mathrm{s}, 0}$ relation defined by the 2997 SMC RR Lyrae variables in our sample:

$K_{\mathrm{s}, 0}=(-3.17 \pm 0.08) \log P+(17.68 \pm 0.02)$

We used the non-weighted fit in order to avoid biasing by brighter objects which usually have smaller uncertainties. The fit is shown in Fig. 6. The r.m.s. of the relation is large $(0.17 \mathrm{mag})$ and could be owing to: metallicity differences, intrinsic dispersion of the $P K_{\mathrm{s}, 0}$ relation or depth effect. The dependence of the $K$-band magnitude on metallicity has been investigated in a number of studies with a tendency of the theoretical and semi-theoretical
Structure of the SMC from RR Lyrae stars

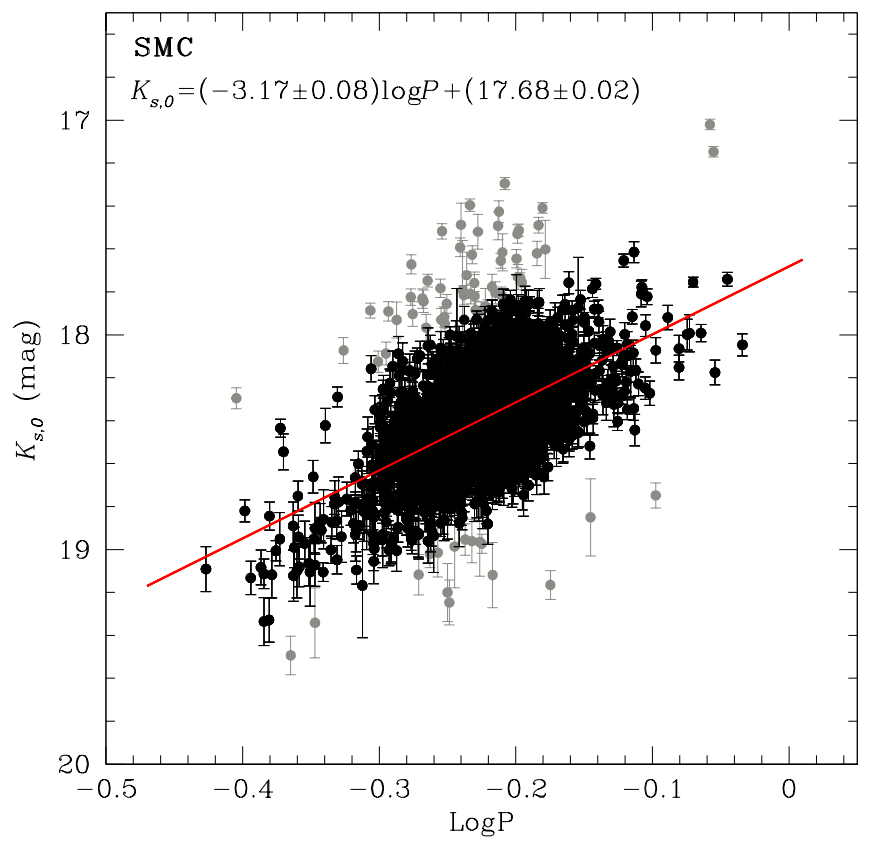

Figure 6. $P K_{\mathrm{s}, 0}$ relation of $2997 \mathrm{RR}$ Lyrae stars in the SMC. The relation was calculated as a non-weighted linear regression by progressively discarding objects which deviate more than $3 \sigma$. Black and grey dots represent objects located within and beyond $3 \sigma$ from the regression line, respectively. The red line shows the best fit.

analyses (Bono et al. 2003; Catelan et al. 2004) to derive a steeper metallicity slope than found by the empirical analyses (Del Principe et al. 2006; Sollima et al. 2006, 2008; Borissova et al. 2009; Muraveva et al. 2015). Literature empirical values for the metallicity slope vary from $0.03 \pm 0.07$ (Muraveva et al. 2015 based on 70 field RR Lyrae variables in the bar of the LMC) to $0.12 \pm 0.04$ (Del Principe et al. 2006 from the analysis of RR Lyrae stars in $\omega$ Cen). Thus, the dependence of the $K_{\mathrm{s}}$ magnitude on metallicity does not seem to be able to explain the large scatter seen in Eq. 5 . Hence, we conclude that the large spread observed in the RR Lyrae $P K_{\mathrm{s}}$ relation is mainly caused by the intrinsic dispersion of the $P K_{\mathrm{s}, 0}$ relation and depth effect. A detailed discussion of the SMC line-of-sight depth and intrinsic dispersion of the $P K_{\mathrm{s}, 0}$ relation is provided in Section 4.2.

To reduce the scatter we calculated the $P K_{\mathrm{s}, 0}$ relations as a non-weighted linear regression by progressively discarding objects that deviate more than $3 \sigma$, in each of the 27 SMC tiles selected for the present analysis, separately. The resulting relations are summarised in column 6 of Table 1 and shown in Fig. 7. The r.m.s. of the relations remains significant (0.13-0.24 mag) even for single tiles and is systematically larger in the inner regions of the SMC. The slope of the $P K_{\mathrm{s}, 0}$ relation in different tiles varies from -1.57 to -4.14. This provides hints of an elongated structure of the SMC, which we studied using individual distances to the 2997 RR Lyrae stars in our sample. 


\section{STRUCTURE OF THE SMC}

\subsection{Individual distances of RR Lyrae stars}

Using the Fourier parameters of OGLE IV light curves Skowron et al. (2016) estimated individual metallicities on the Jurcsik (1995) and Zinn \& West (1984) metallicity scales for 3560 RRab stars in the SMC. We found that 2426 out of 2997 RR Lyrae stars in our sample have individual metallicities estimated by Skowron et al. (2016). We used the metallicity estimates on the Zinn \& West metallicity scale from Skowron et al. (2016) for 2426 RR Lyrae stars in the sample, and to the 571 RR Lyrae stars without an individual metallicity estimate we assigned the mean metallicity of the SMC RRab stars determined by the same authors: $[\mathrm{Fe} / \mathrm{H}]_{\mathrm{ZW} 84}=-1.85 \pm 0.33$ dex. These 571 stars are distributed smoothly in the SMC with a slight concentration in the bar-like feature, where the crowding could have prevented the accurate estimation of metallicity.

We use our dereddened intensity-averaged $K_{\mathrm{s}, 0}$ magnitudes (Section 2.3), periods from the OGLE IV catalogue (Soszyński et al. 2016) and metallicities from Skowron et al. (2016) along with eq. 16 from Muraveva et al. (2015) to determine distance moduli of the 2997 SMC RR Lyrae stars. The $P M_{K_{\mathrm{s}}} Z$ relation in Muraveva et al. (2015) was obtained on the metallicity scale defined in Gratton et al. (2004). Gratton et al. (2004) pointed out that there is no clear offset between the metallicity scales defined in their paper and Zinn \& West (1984) metallicity scale, and considered the 0.06 dex difference found for the three calibrating clusters used in their analysis as a possible offset. Hence, we added 0.06 dex to the metallicity values provided by Skowron et al. (2016). However, the dependence on metallicity of the $M_{K_{\mathrm{s}}}$ magnitudes is small (0.03, Muraveva et al. 2015), hence, a 0.06 dex offset will give only 0.002 mag error to the distance modulus, which is much smaller than typical errors of distance moduli $(0.15 \mathrm{mag})$. Thus, the uncertainty in the offset between two metallicity scales will not affect our final results. Individual distance moduli obtained for $2997 \mathrm{RR}$ Lyrae stars in the sample are summarised in column 11 of Table 3.

The weighted mean distance modulus of the $2997 \mathrm{RR}$ Lyrae stars in our sample is $(m-M)_{0}=18.88 \mathrm{mag}$, with a standard deviation of $0.20 \mathrm{mag}$. This is $0.08 \mathrm{mag}$ shorter than derived by de Grijs \& Bono (2015) based on a statistical analysis of the SMC distance estimates available in the literature, $(m-M)_{0}=18.96 \pm 0.02 \mathrm{mag}$, but both determinations are entirely consistent with each other within the mutual uncertainties (standard deviations). It is also shorter than the estimates of the SMC's distance modulus based on Classical Cepheids observed by VMC $(m-M)_{0}=19.01 \pm 0.05 \mathrm{mag},(m-M)_{0}=19.04 \pm 0.06 \mathrm{mag}$ (Ripepi et al. 2016) and $(m-M)_{0}=19.01 \pm 0.08 \mathrm{mag}$ (Marconi et al. 2017). Note that the mean distance modulus derived in the current study reflects only the statistical distribution of individual distance moduli in the sample, and is not a distance to the centre of the ellipsoid formed by RR Lyrae stars. The derived mean value is significantly affected by the spatial distribution of RR Lyrae stars and cannot be considered as a distance to the SMC. Distance modulus derived in the current study corresponds to the mean distance $60.0 \mathrm{kpc}$. The standard deviation of the mean value is $\sim 5$ $\mathrm{kpc}$ and reflects the extension of the SMC RR Lyrae star distribution along the line-of sight (see Section 4.2).

We also calculated mean distances to the RR Lyrae stars in each of the 27 tiles, separately (Table 1 ). The derived distance moduli span the range from 18.78 to 18.94 mag. There is a clear trend of tiles located in the eastern and south-eastern parts of the SMC to be closer to us. However, the distances to the stars in a given tile calculated in this way are approximate, since the same distance is assigned to all stars in the tile. To perform a more accurate analysis of the SMC's structure we used the individual distances of the 2997 RR Lyrae variables. In Fig. 8 we present the distance modulus distributions defined by the individual $R R$ Lyrae stars in each tile. It shows that the eastern regions have asymmetric distributions of the RR Lyrae stars and are located closer to us.

The two-dimensional distribution of distances in the $\mathrm{SMC}$ is shown in Fig 9. The upper-left, upper-right, lowerleft and lower-right panels show respectively the closer RR Lyrae stars with $(m-M)_{0}<18.68 \mathrm{mag}$, the more distant RR Lyrae stars with $(m-M)_{0}>19.08$ mag, the sample within $1 \sigma$ error of the mean distance modulus and the total sample. RR Lyrae stars at all distances are distributed smoothly. We cannot see any signature of the bar from the distribution of RR Lyrae stars.

Similarly to Fig. 9, in Fig. 10 we plot the twodimensional distribution of extinction, $A_{K_{\mathrm{s}}}$, derived in Section 2.3. The upper-left and upper-right panels of Fig. 10 show that more distant RR Lyrae stars in general have higher values of extinction than closer stars. The mean extinction of the closest $\mathrm{RR}$ Lyrae variables is $\left\langle A_{K_{\mathrm{s}}}\right\rangle=$ 0.017 mag which should be compared with $\left\langle A_{K_{\mathrm{s}}}\right\rangle=$ $0.023 \mathrm{mag}$ derived for the most distant stars. The highest extinction is observed in the central part and in the active star-forming region in an eastern extension of the SMC called the Wing, located at $\alpha=01^{\mathrm{h}} 15^{\mathrm{m}}$ and $\delta=$ $-73^{\circ} 10^{\prime}$ (Haschke et al. 2011). The concentration of stars with high extinction in the central regions seen in all panels of Fig. 10 extends from the north-east to south-west and outlines the bar-like feature of the SMC. Thus, although we could not detect the bar from the spatial distribution of RR Lyrae stars (Fig. 9), the bar-like feature is seen in the two-dimensional distribution of the extinction (Fig. 10). Note that a higher extinction in the Wing of the SMC is seen for all RR Lyrae stars except the closest ones, which could mean that the star-forming region is located at a greater distance than $(m-M)_{0}=18.68 \mathrm{mag}$. These findings are in agreement with results obtained by Haschke et al. (2011) and Subramanian \& Subramaniam (2012) who measured the reddening in the SMC using RC stars detected by OGLE III and found the main concentrations of higher reddening in the bar and Wing of the SMC. Deb (2017) determined individual reddening for OGLE IV RR Lyrae stars and found that the south-eastern part of the SMC has the regions of the higher extinction, which is in agreement with our findings.

We calculated the centroid of our sample as an average of RA and Dec values of the RR Lyrae stars, and found that it is located at $\alpha_{0}=00^{\mathrm{h}} 55^{\mathrm{m}} 50^{\mathrm{s}} .97$ and $\delta_{0}=-72^{\circ} 51^{\prime} 29^{\prime \prime} .27$. We divided the whole sample of RR Lyrae stars according to $\mathrm{RA}$ in an eastern $\left(\mathrm{RA}>\alpha_{0}\right)$ and a western $\left(\mathrm{RA}<\alpha_{0}\right)$ region based on their positions. There are 1473 and 1524 RR Lyrae 
stars in the eastern and the western regions, respectively. The distance modulus distributions (for a bin size of 0.05 mag) of the RR Lyrae variables in the eastern (blue line) and western (red dotted line) regions of the SMC are shown in Fig. 11. The two distributions show a difference with an excess (15\%) of closer RR Lyrae variables in the eastern region. We note that the error in individual distances has a range from 0.1 to $0.2 \mathrm{mag}$, with an average error of $0.15 \mathrm{mag}$. This is larger than the bin size used in Fig. 11. We changed the bin sizes from 0.05 to $0.2 \mathrm{mag}$ and we do observe an excess of $\sim 15 \%$ closer RR Lyrae stars in the eastern regions for all bin sizes.

The mean distances estimated to the eastern and western regions of the SMC are, respectively, $59.56 \pm 0.14 \mathrm{kpc}$ and $60.50 \pm 0.14 \mathrm{kpc}$, where errors are calculated as standard deviation divided by square root of number of stars in the region. This suggests that the eastern region is $\sim$ $0.94 \pm 0.20 \mathrm{kpc}$ closer to us than the western region. We considered whether this asymmetry could be caused by an overestimation of the extinction in the eastern region. In order to check this statement we assigned the mean value of extinction derived in Section $2.3\left\langle A_{K_{\mathrm{s}}}\right\rangle=0.02 \mathrm{mag}$ to all 2997 stars in the sample and derived mean distances to the eastern and western regions of the SMC of $59.57 \pm 0.14 \mathrm{kpc}$ and $60.40 \pm 0.14 \mathrm{kpc}$, respectively. These distances are in agreement with the values obtained by applying individual extinction corrections and proves that the distance determination from the $K_{\mathrm{s}}$ magnitudes is not affected by a variation of extinction in the SMC. Furthermore, there is still a difference of $0.83 \pm 0.20 \mathrm{kpc}$ between the mean distances of RR Lyrae stars in the eastern and western regions. Thus, the appearance of the eastern region closer to us is not owing to an overestimation of the extinction and rather reflects the actual spatial distribution of the SMC RR Lyrae stars. This is consistent with results obtained by Subramanian \& Subramaniam (2012), Haschke et al. (2012) and Deb et al. (2015) from the study of SMC RR Lyrae stars using the OGLE III data. Recent results from Jacyszyn-Dobrzeniecka et al. (2016) based on RR Lyrae stars using the OGLE IV catalogue do not imply any sub-structures but show some asymmetry in the equal density contours in the eastern part of the SMC. They detect a shift in the centre of the ellipsoidal fit, to RR Lyrae stars in different density bins, towards the east and closer to the observer as a function of radius from the SMC centre. This again suggests that the eastern region of the SMC includes a larger number of closer RR Lyrae stars in the entire OGLE IV data, in agreement with our results.

\subsection{Mean distances and line-of-sight depth}

The variation of the line-of-sight depth across the SMC can be obtained by measuring the mean distance and standard deviation in smaller sub-regions. We divided the observed SMC region into smaller sub-regions of equal area $(0.6 \times$ $0.5 \mathrm{deg}^{2}$ ), as anticipated in Section 2.3 (110 sub-regions in total). The standard deviation with respect to the mean distance is a measure of the line-of-sight depth. Along with the actual line-of-sight depth in the distribution, the standard deviation has contributions from the intrinsic magnitude spread of RR Lyrae stars owing to errors in photometry and metallicity, as well as the intrinsic luminosity variation owing to evolutionary effects.

To perform an approximate estimation of the intrinsic magnitude spread we analysed the SMC globular cluster NGC 121. This is the oldest SMC cluster. It contains four RR Lyrae stars (Walker \& Mack 1988), which were confirmed to be cluster's members based on their location close to the cluster's centre and their distribution on the Horizontal Branch of the cluster's CMD (Clementini, private communication). As part of our analysis we derived dereddened $K_{\mathrm{s}, 0}$ magnitudes for three of them: V32, V35 and V37 (identification from Walker \& Mack 1988). The RR Lyrae star V36 was not included in our analysis since it turned out to be an RRc star according to the OGLE IV catalogue (Soszyński et al. 2016). We used the $K_{\mathrm{s}, 0}$ magnitudes from VMC, periods from the OGLE IV catalogue, the metallicity value of $[\mathrm{Fe} / \mathrm{H}]=-1.51 \mathrm{dex}$ (Zinn \& West 1984) and eq. 16 from Muraveva et al. (2015) to determine individual distance moduli of the RR Lyrae stars in NGC 121. The mean distance modulus of NGC 121 from the RR Lyrae stars is $(m-M)_{0}=18.97 \pm 0.07 \mathrm{mag}$. Since the three variables belong to the same cluster we consider the depth effect negligible, hence, the standard deviation of the mean value, $0.07 \mathrm{mag}$, represents the intrinsic magnitude spread caused by the effects described above. Clementini et al. (2003) estimated the intrinsic $V$-magnitude spread of $101 \mathrm{RR}$ Lyrae stars in the LMC caused by the internal photometric errors, metallicity distribution and evolutionary effect as 0.10 mag. Since the dependence of the $K_{\mathrm{s}}$ magnitudes on metallicity and evolutionary effect is smaller than in $V$ band, our estimation of intrinsic dispersion in the SMC as $0.07 \mathrm{mag}$ is reasonable, even though it is based only on three stars.

The actual line-of-sight depth in the 110 SMC subregions is estimated as $\sigma_{\text {los }}^{2}=\sigma_{\text {measured }}^{2}-\sigma_{\text {intrinsic }}^{2}$. Figs. 12 and 13 show the two-dimensional distributions we obtain for mean distance and actual line-of-sight depth, respectively. The mean distance map clearly shows that the eastern subregions are closer to us, as it was widely discussed above. The actual line-of-sight depth values range from 1 to $10 \mathrm{kpc}$, with an average depth of $4.3 \pm 1.0 \mathrm{kpc}$. Fig. 13 shows that the largest depth is found in the central regions of the SMC. The standard deviation associated with the mean distance modulus of our entire sample is $0.2 \mathrm{mag}$ (Section 4.1). After correcting for the intrinsic width, this dispersion corresponds to a line-of-sight depth of $5.2 \mathrm{kpc}$.

The tidal radius of the SMC is estimated as $4-9 \mathrm{kpc}$ by Stanimirović, Staveley-Smith \& Jones (2004), 7 - 12 kpc by Subramanian \& Subramaniam (2012) and De Propris et al. (2010) estimated the SMC edge in the eastern direction to be at a radius of $6 \mathrm{kpc}$. Nidever et al. (2011) found the presence of old/intermediate-age populations at least up to a radius of $9 \mathrm{kpc}$. These values are comparable to the $1 \sigma$ depth of 10 kpc observed in the central regions of the SMC. 

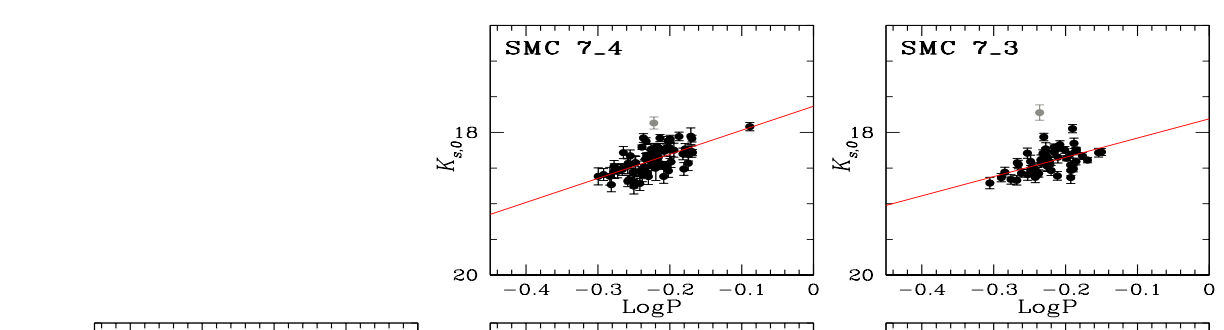

$\varpi$
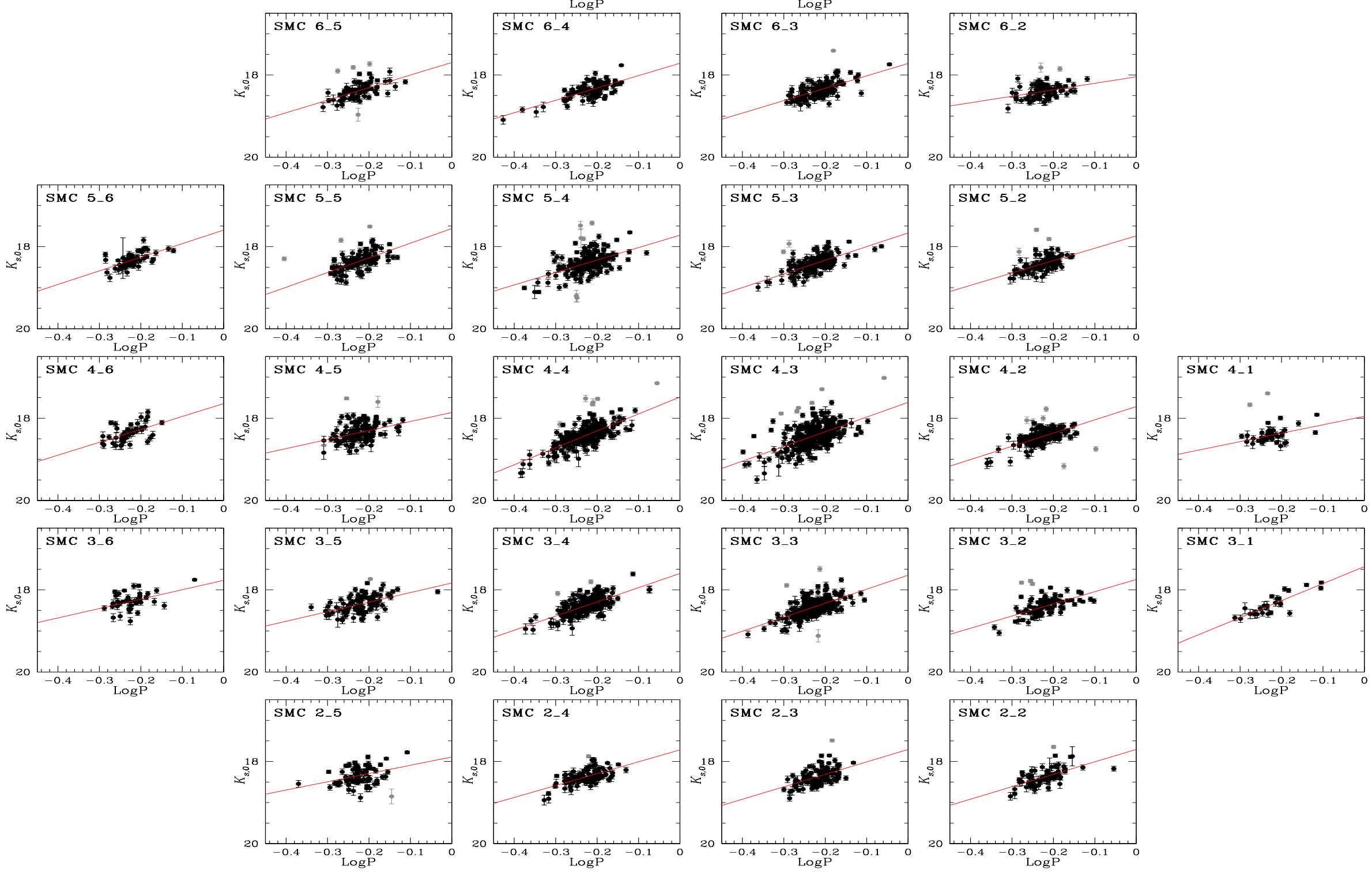

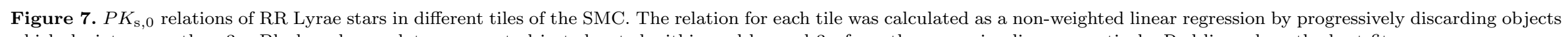

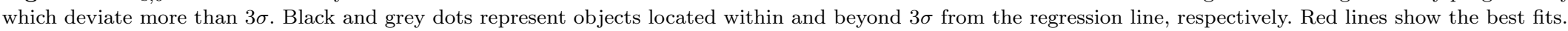
Downloaded from https://academic.oup.com/mnras/article-abstract/doi/10.1093/mnras/stx2514/4259591/The-VMC-survey-XXVI-Structure-of-the-Small 

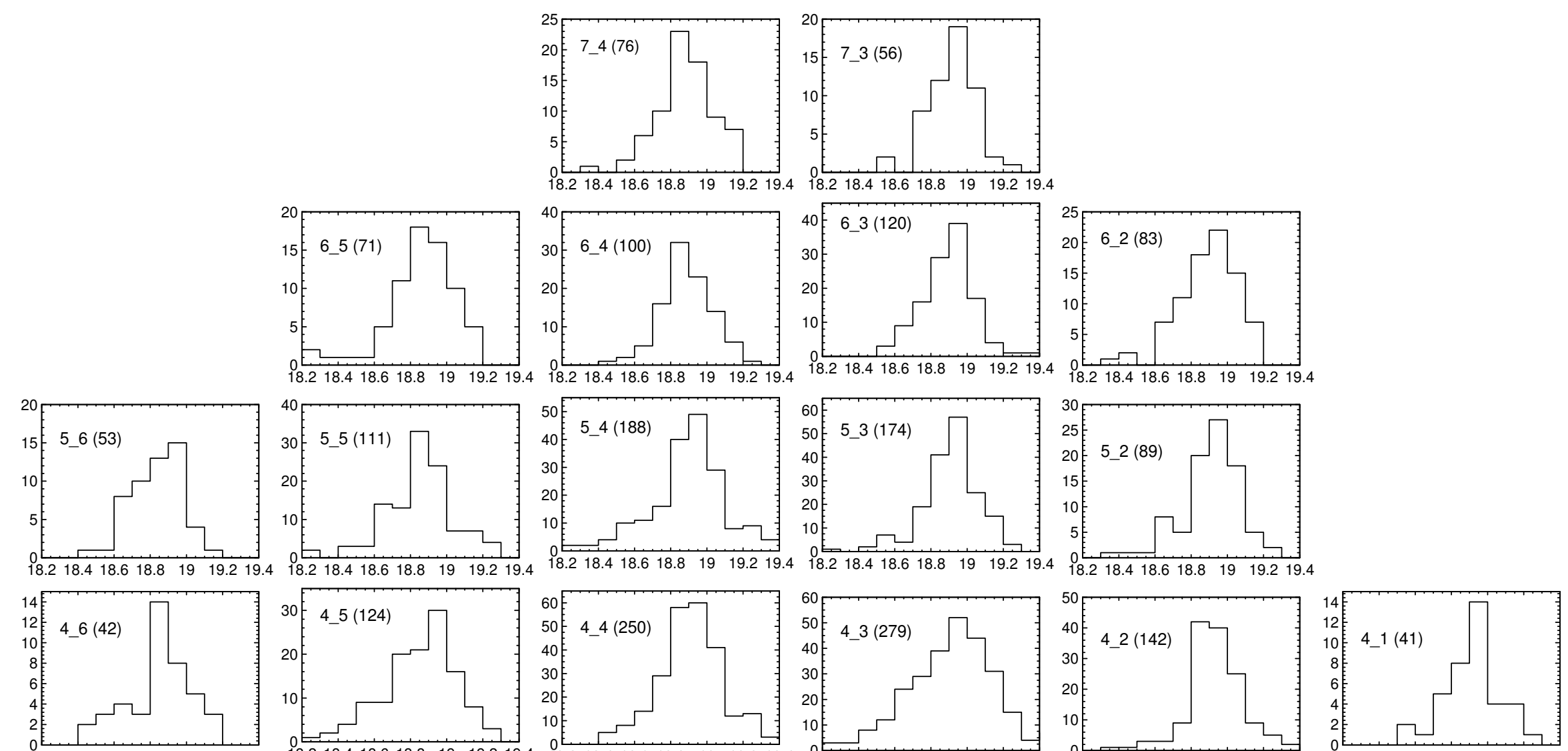

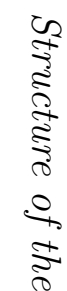
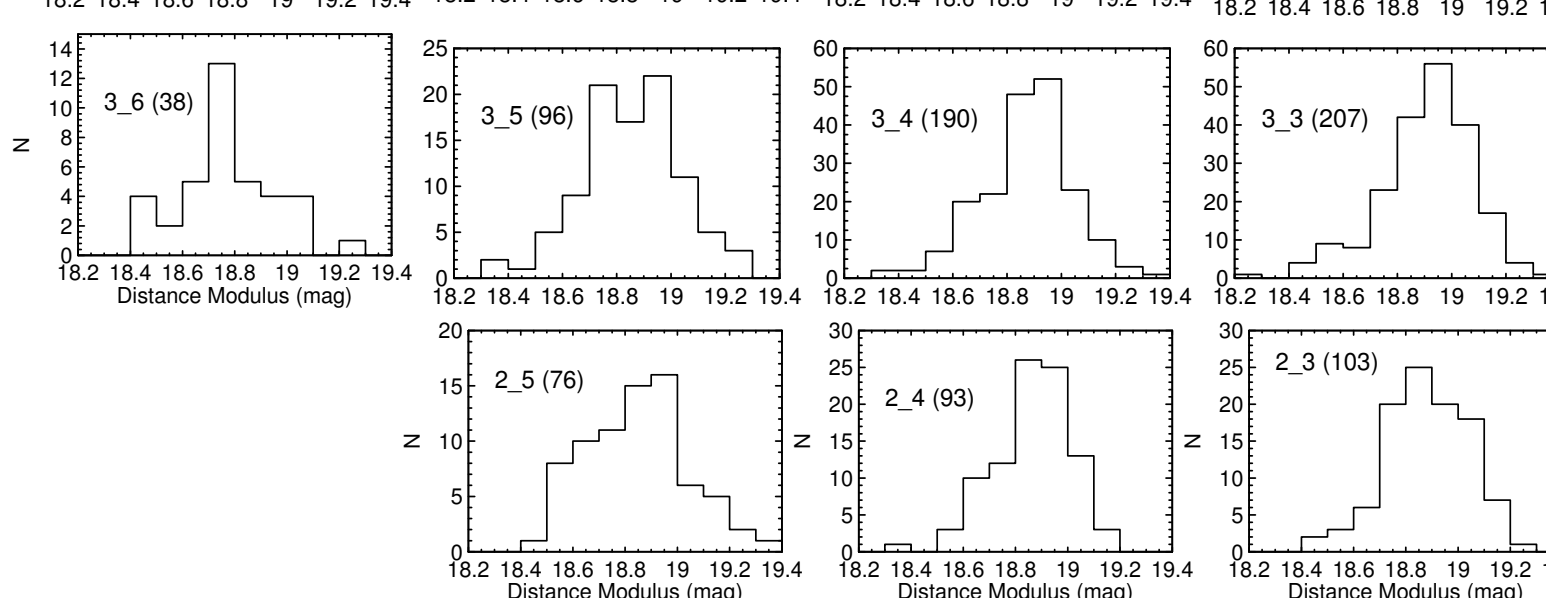

Distance Modulus (mag)

$8.418 .618 .8 \quad 19 \quad 19.2$
Distance Modulus (mag)
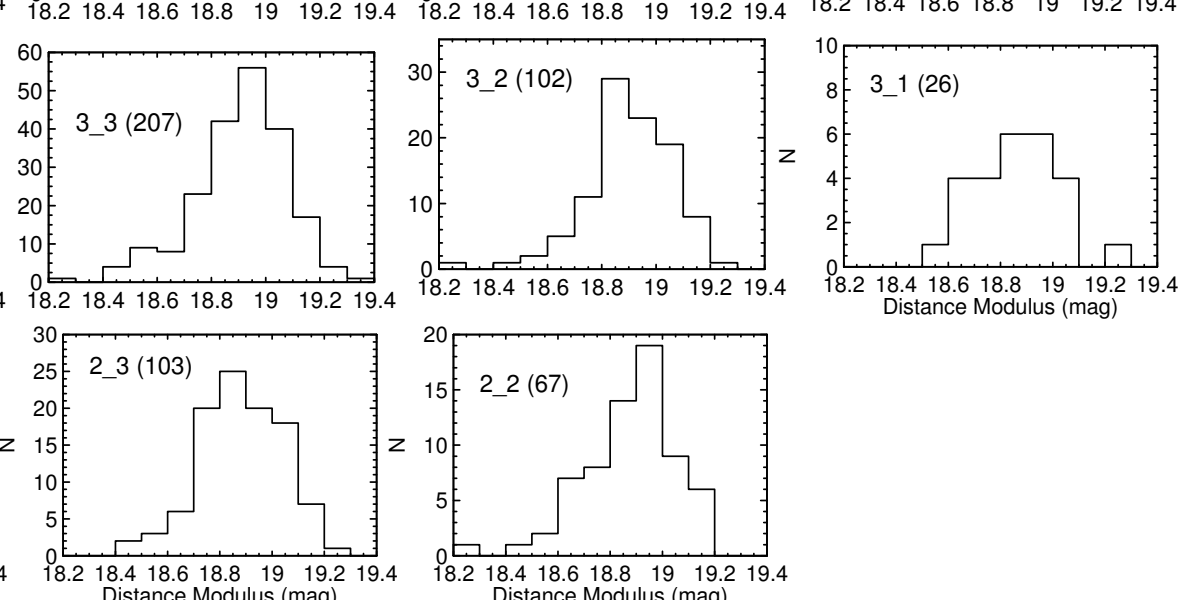

18.418 .618 .819 19.2
Distance Modulus (mag)

Distance Modulus (mag)

Distance Modulus (mag)

Figure 8. Distance modulus distributions of the RR Lyrae stars in different tiles. The tile number is shown without SMC in prefix. The total number of RR Lyrae stars in each tile is shown in parenthesis. 


\subsection{Three dimensional structure of the SMC}

The Cartesian coordinates corresponding to each RR Lyrae star can be obtained using the star's RA, Dec coordinates and the distance modulus. We assume the $x$-axis antiparallel to the RA axis, the $y$-axis parallel to the Dec axis, and the $z$-axis along the line-of-sight with values increasing towards the observer. The distance modulus is used to obtain the distance to each star in kpc. The RA, Dec and the distance are converted into $x, y, z$ Cartesian coordinates using the transformation equations given by van der Marel \& Cioni (2001) and assuming the origin of the system at $\alpha_{0}=00^{\mathrm{h}} 55^{\mathrm{m}} 50^{\mathrm{s}} .97$ and $\delta_{0}=-72^{\circ} 51^{\prime} 29^{\prime \prime} 27$. The distance to the origin of the $\mathrm{SMC}$ is taken as $60 \mathrm{kpc}$ with standard deviation of $5 \mathrm{kpc}$ (Section 4.1). RA and Dec of each star correspond to the VMC coordinates. The positional accuracy of the VMC survey is $\sim 0.02^{\prime \prime}$. The errors associated with $x, y$ and $z$ are calculated using error propagation.

Based on both observations and theoretical studies (Subramanian \& Subramaniam 2012 and references therein), the old and intermediate-age stellar populations in the SMC are suggested to be distributed in a spheroidal/ellipsoidal system. Our sample of SMC RR Lyrae stars shows a smooth distribution on the sky. Thus we modelled the RR Lyrae stellar distribution as a triaxial ellipsoid. The parameters of the ellipsoid, such as the axes ratio, the position angle of the major axis of the ellipsoid projection on the sky $(\phi)$ and the inclination of the longest axis with respect to the sky plane $(i)$ are estimated using a method of inertia tensor analysis similar to that described in Subramanian \& Subramaniam (2012) (also refer to Pejcha \& Stanek 2009; Paz et al. 2006). The basic principle is to create an inertia tensor of the $x, y, z$ coordinates and estimate the Eigen vectors and Eigen values. The Eigen vectors correspond to the spatial directions and the square roots of the Eigen values correspond to the axes ratio.

First, we applied the method to the $(x, y)$ coordinates and found that the stars are located in an elongated distribution with an axes ratio of $1: 1.12( \pm 0.01)$ and the major axis has a position angle $\phi=85^{\circ} .5 \pm 0^{\circ} .1$. We repeated the procedure with $(x, y, z)$ coordinates. The $z$ values have a large scatter, owing to large uncertainty in distances, compared to good positional accuracy $\left(0.02^{\prime \prime}\right)$. As described in Pejcha \& Stanek (2009), to correct for this internal scatter in the $z$ value, we subtracted the internal scatter of 0.07 mag from the z-component (see Section 4.2). We obtained axes ratio, $\phi$ and $i$ values of $1: 1.11( \pm 0.01): 3.30( \pm 0.70)$, $84^{\circ} .6 \pm 0^{\circ} .2$ and $2^{\circ} .1 \pm 0^{\circ} .6$, respectively. The small inclination angle of the longest axis with respect to the sky plane suggests that the longest axis of the ellipsoidal distribution of the RR Lyrae stars is oriented nearly along the line-ofsight.

A compilation of structural parameters of the SMC ellipsoid from the RR Lyrae stars available in the literature is provided in Table 2. In the majority of these studies the RR Lyrae stars from the OGLE III catalogue were used, while Jacyszyn-Dobrzeniecka et al. (2016) analysed RRab stars in the OGLE IV catalogue, which covers almost entirely the SMC. In the current study we used RR Lyrae variables observed by OGLE IV which also have counterparts in the VMC catalogue. Qualitatively all previous results indicate that the RR Lyrae stars in the SMC are distributed in an ellipsoid with the longest axis oriented almost along the lineof-sight. Our results also support these findings, but quantitatively there are some differences. This could be mainly owing to the difference in the spatial coverage of the data used in the various studies. Fig. 14 shows the distribution of RRab stars used in the this paper and RRab stars from the OGLE III and OGLE IV catalogues. OGLE IV covers larger area than VMC and OGLE III, which are basically focused on the central regions of the SMC, where majority of RR Lyrae stars are concentrated.

\subsection{Effect of interaction of the Magellanic Clouds}

Mutual interactions between the Magellanic Clouds and the resultant tidal stripping of material from the SMC are believed to be the most probable scenario for the formation of the MB (Diaz \& Bekki 2012; Besla et al. 2012). One of the challenges to this scenario is the lack of conclusive evidence for the presence of tidally stripped intermediateage/old stars in the MB. Nidever et al. (2013) identified a closer (distance, $\mathrm{D} \sim 55 \mathrm{kpc}$ ) stellar structure in front of the main body of the eastern SMC, which is located $4^{\circ}$ from the SMC centre. These authors suggested that it is the tidally stripped stellar counterpart of the $\mathrm{H} \mathrm{I}$ in the MB. Subramanian et al. (2017) also identified a foreground population (at $\sim 12 \pm 2 \mathrm{kpc}$ in front of the main body of the SMC), whose most likely explanation is tidal stripping from the SMC. Moreover, they identified the inner region (at $~$ $2-2.5 \mathrm{kpc}$ from the centre) from where the signatures of interactions start becoming evident, thus, supporting the hypothesis that the MB was formed from tidally stripped material from the SMC. Both these studies were based on RC stars. Recently, Ripepi et al. (2017) found that the young and old Cepheids in the SMC have different geometric distributions. They also found closer Cepheids in the eastern regions which are off-centred in the direction of the LMC. They suggest that these results are owing to the tidal interaction between the Magellanic Clouds.

The RR Lyrae stars which are older (age $\geq 10$ Gyr) than RC stars (age $\sim 2-9$ Gyr) and Cepheids (100-300 Myr) are also expected to be affected by tidal interactions. The RR Lyrae stars are well identified and we have accurate distances to these individual stars. The presence of tidal signatures in the oldest populations will provide strong constraints to theoretical models which explain the formation of the MS. Hence, we explore our data set in more detail.

In the present study we see a shift in the mean distance of the RR Lyrae stars in the eastern region compared to the western region. We also see that RR Lyrae stars in the eastern tiles have asymmetric distance distributions (Fig. 8) and the mean distances of the eastern sub-regions are shorter (Fig. 12). Fig. 15 presents the radial variation of the mean distance to the various sub-regions. The blue hexagons represent sub-regions in the eastern and the red triangles represent sub-regions in the western parts of the SMC. The plot shows that the eastern sub-regions are located at shorter distances compared to the western sub-regions. Beyond $2^{\circ}$ in radius from the centre, the majority of the eastern subregions have shorter distances. This angular radius corresponds to a linear radius of $\sim 2.2 \mathrm{kpc}$ from the SMC centre and is similar to the radius from where the signatures of interactions, in the form of bimodality in the distribution 

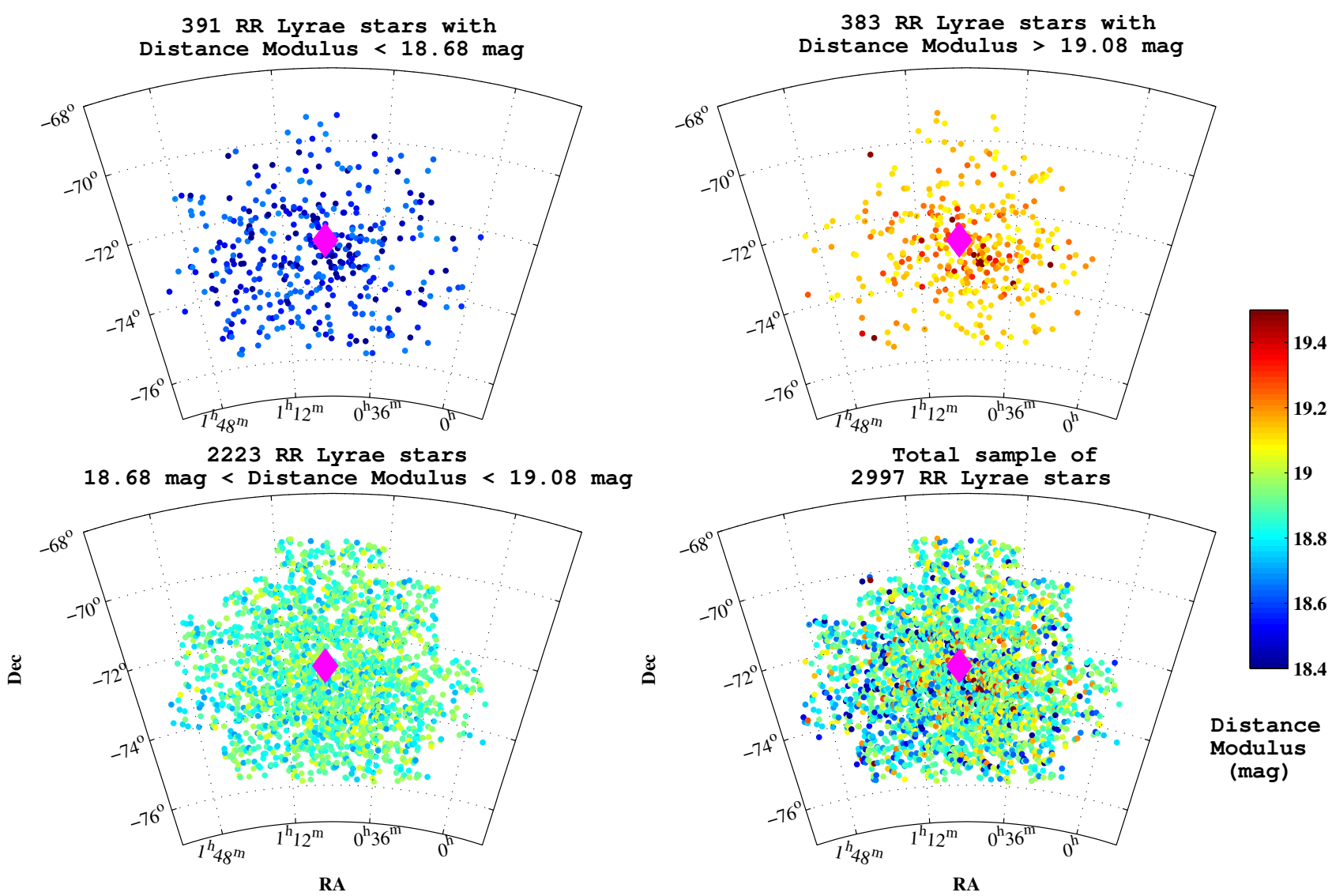

Figure 9. Two-dimensional distribution of the RR Lyrae stars' distance moduli. The upper-left, upper-right, lower-left and lower-right panels show respectively the closer RR Lyrae stars with $(m-M)_{0}<18.68 \mathrm{mag}$, the more distant RR Lyrae stars with $(m-M)_{0}>$ $19.08 \mathrm{mag}$, the sample within $1 \sigma$ error of the mean distance modulus and the total sample. The magenta diamond in all panels represents the centroid of the sample.

Table 2. Structural parameters of the SMC ellipsoid as inferred from the RR Lyrae stars.

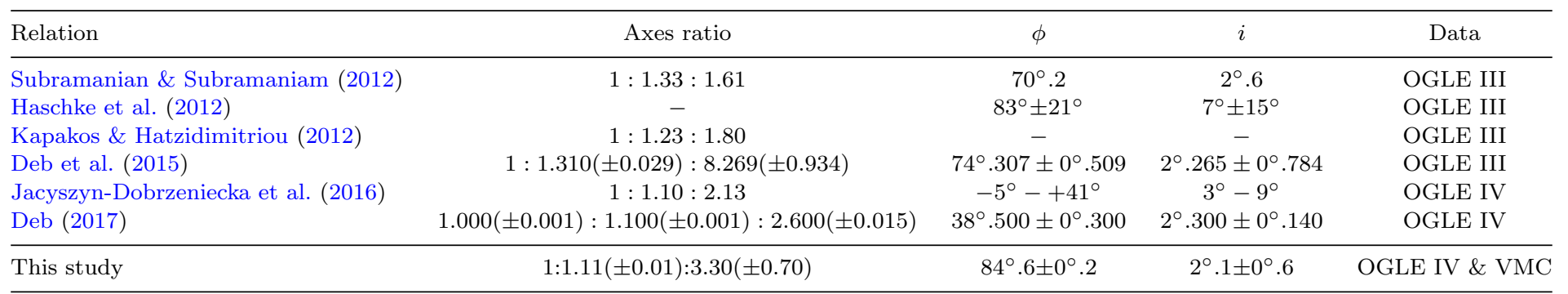

of RC stars, start to become evident (Subramanian et al. 2017). We point out that the typical error associated with the mean distances of the sub-regions is $\sim 1 \mathrm{kpc}$ (as shown in the top-right of the plot). Thus, our results indicate that the oldest stellar population in the eastern part of the SMC, in the direction of the MB, is affected by the interactions of the Magellanic Clouds, 200-300 Myr ago, which is believed to be mainly responsible for the formation of the MB.

However, in our present study using RR Lyrae stars, we do not see a clear bimodality as observed between the two peaks of the RC distribution (Subramanian et al. 2017, two peaks separated by $\sim 12 \pm 2 \mathrm{kpc}$ ) in the eastern part of the SMC. While comparing our Fig. 8 with fig. 6 of Subramanian et al. (2017), the discrepancy is very evident for tiles 6_5 and 5_6. This implies that the RC stars and RR Lyrae stars are distributed in a different structural components in the eastern SMC and/or a different origin of the foreground RC population. Subramanian et al. (2017) presented the results for $13 \mathrm{VMC}$ tiles covering the entire central part and some outer regions. We plan to address the observed discrepancy between the RC and RR Lyrae stars distributions in our future work based on the analysis of the double RC feature in all the SMC tiles observed by VMC.

Recently, Belokurov et al. (2017) identified tidal tails 

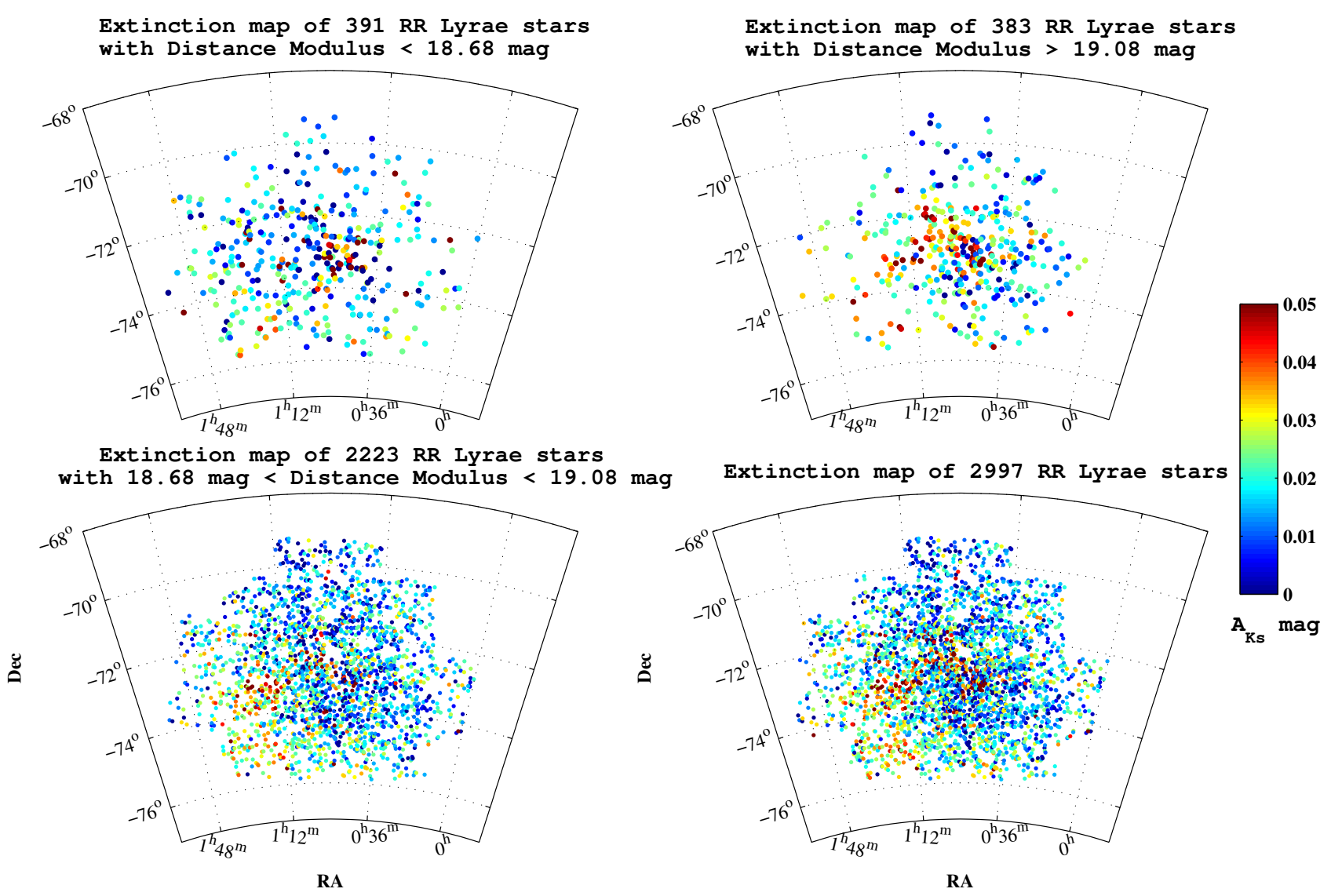

Figure 10. Two-dimensional distribution of the RR Lyrae stars' extinction $A_{K_{\mathrm{S}}}$. The upper-left, upper-right, lower-left and lower-right panels show respectively the closer RR Lyrae stars with $(m-M)_{0}<18.68 \mathrm{mag}$, the more distant RR Lyrae stars with $(m-M)_{0}>$ $19.08 \mathrm{mag}$, the sample within $1 \sigma$ error of the mean distance modulus and the total sample.

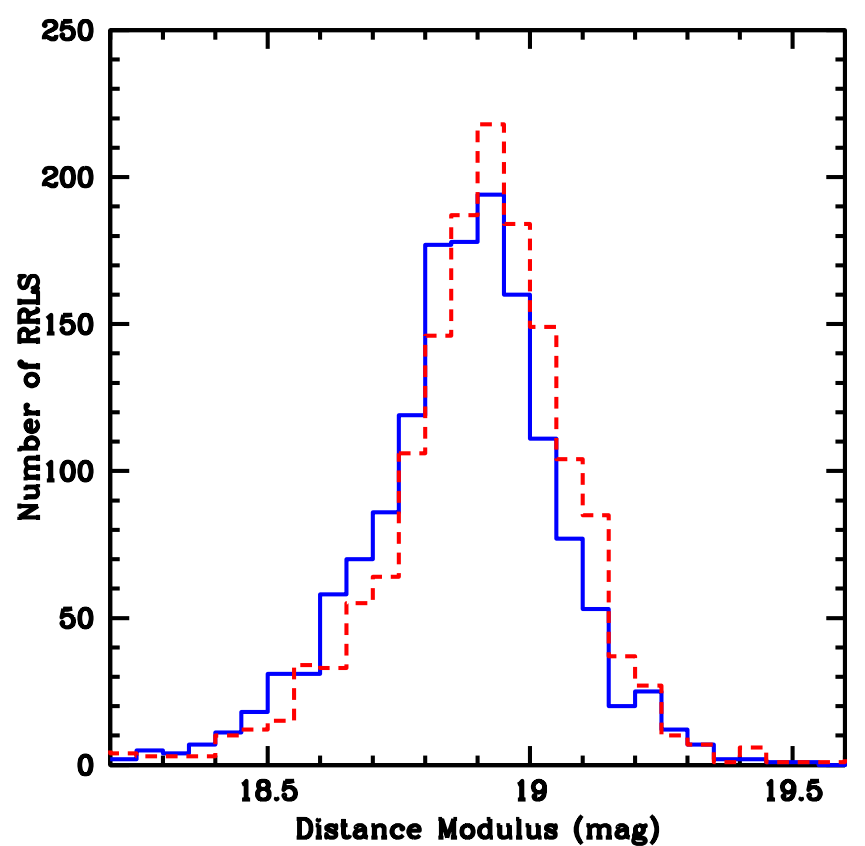

Figure 11. Normalised distance distributions of the RR Lyrae stars in the eastern (blue line) and western (red line) regions of the SMC.

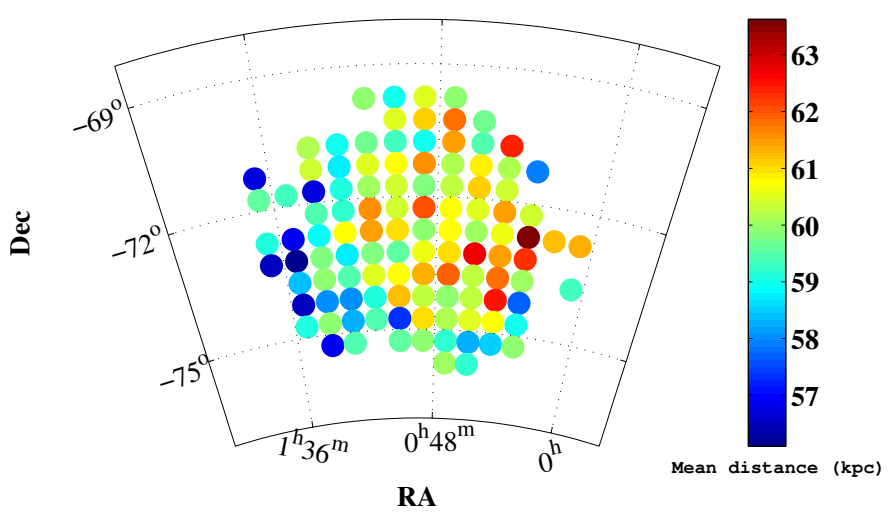

Figure 12. Two-dimensional distribution of the mean distance measured in different sub-regions of the SMC. Each point corresponds to a sub-region of $0.6 \times 0.5 \mathrm{deg}^{2}$ area, for a total number of 110 sub-regions.

around the LMC and the SMC using Gaia DR1 (Gaia Collaboration et al. 2016a,b). They found that the SMC's outer stellar density contours show a characteristic S-shape which is a typical signature of tidal stripping seen 


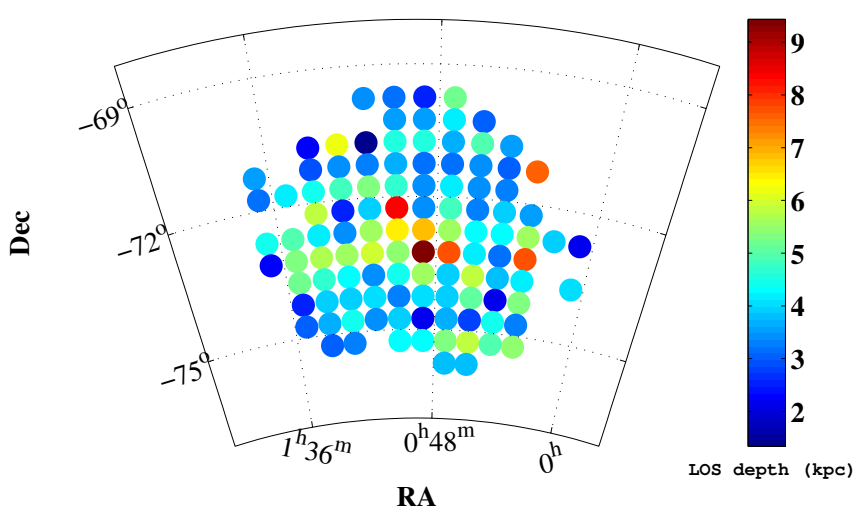

Figure 13. Two-dimensional distribution of the line-of-sight depth measured in different sub-regions of the SMC. Each point corresponds to a sub-region of $0.6 \times 0.5 \mathrm{deg}^{2}$ area, for a total number of 110 sub-regions.

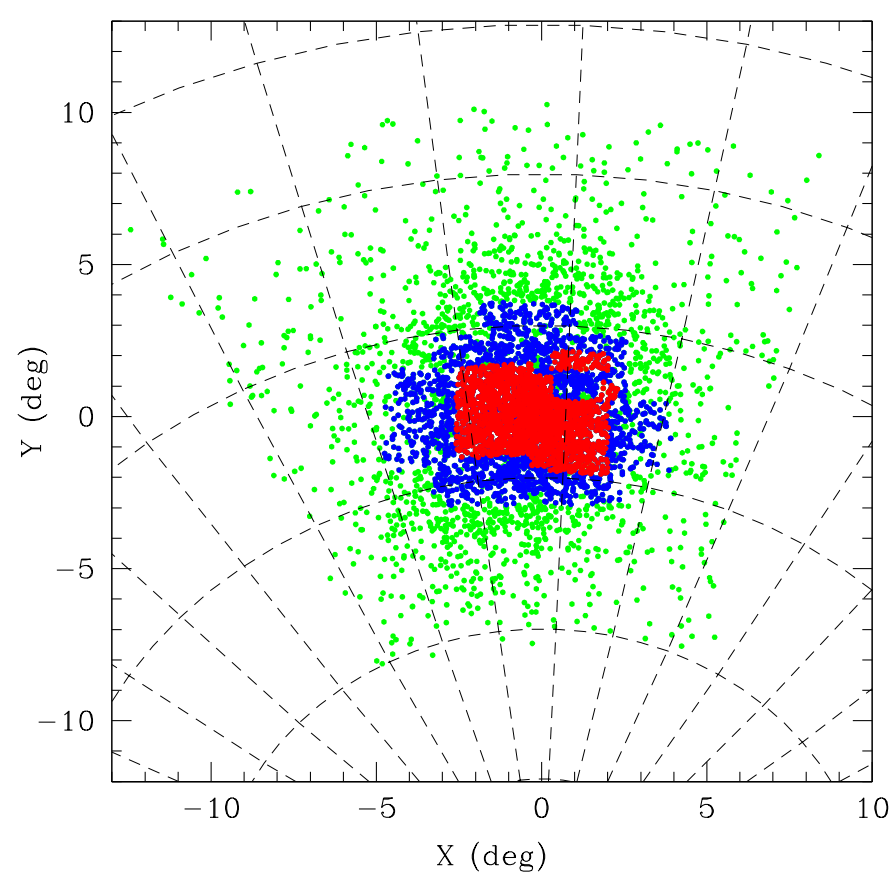

Figure 14. Distribution of RRab stars from the OGLE IV (green dots) and OGLE III (red dots) catalogues, and RRab stars used in the current analysis (blue dots). Coordinates are defined as in van der Marel \& Cioni (2001) where $\alpha_{0}=12.5 \mathrm{deg}, \delta_{0}=-73$ deg.

in satellite galaxies. They also identified a trailing arm (motion of the SMC with respect to the LMC) from the SMC which extends towards the LMC. This stellar tidal tail contains candidate RR Lyrae stars off-set by $\sim 5$ deg from the gaseous MB. Both the old and young MB components originate in the south-eastern part of the SMC $(\sim 6$ deg east and $3 \mathrm{deg}$ south from the SMC centre), where the authors clearly see the trailing tail. Though our observed tiles do not cover the region where Belokurov et al. (2017) identified the SMC's trailing arm, the eastern and south-eastern regions

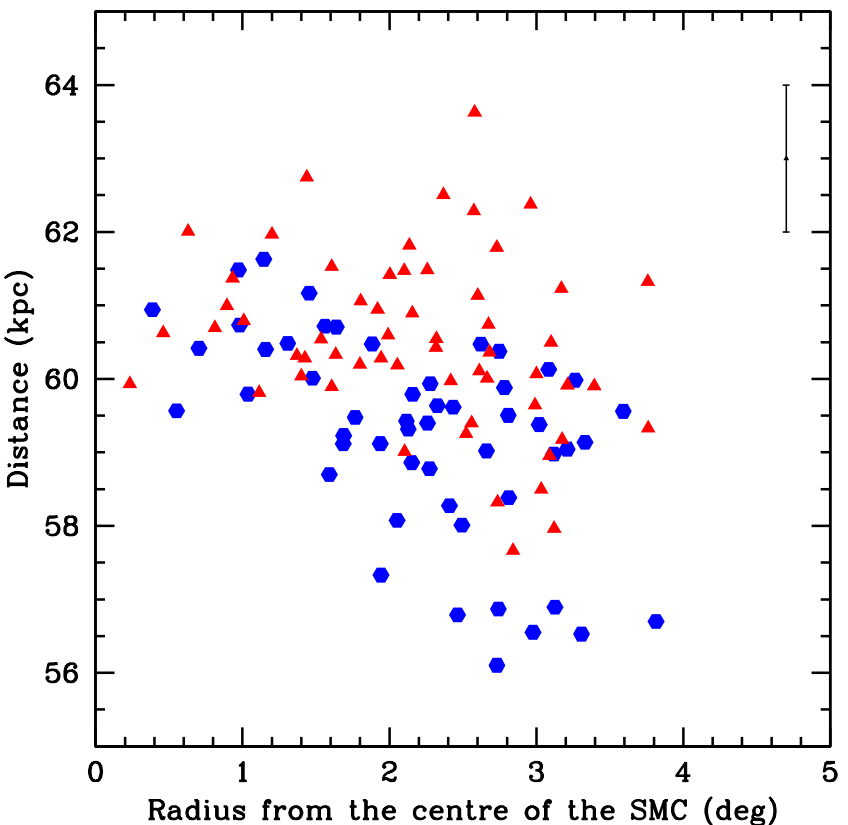

Figure 15. Radial variation of the mean distance of the subregions. Blue hexagons represent regions in the eastern and red triangles represent regions in the western parts, respectively. The typical error bar associated with the mean distance is shown in black on the top-right of the panel.

where we see closer RR Lyrae stars are in the direction of the newly identified SMC's trailing arm.

The study of different stellar populations including RR Lyrae stars in the outer VMC tiles will be interesting to understand this newly identified tidal signature. According to the simulations, the line-of-sight velocities of the stellar debris from the SMC and the LMC are significantly different, hence, a detailed spectroscopic study of RR Lyrae stars and other distance indicators in the eastern regions of the $\mathrm{SMC}$ will provide valuable information to understand the interaction history of the MS.

\section{CONCLUSIONS}

In this study we analysed the structure of the SMC for the first time using multi-epoch near-infrared photometry of RR Lyrae stars observed by the VMC survey. For this analysis 2997 RRab stars distributed in 27 VMC tiles with visual photometry and pulsation periods from the OGLE IV survey, were selected. The well-sampled light curves in the $K_{\mathrm{s}}$ band allowed us to derive accurate intensity-averaged magnitudes for each RR Lyrae star in the sample. Individual reddening values were calculated using the $V$ and $I$ magnitudes from the OGLE IV survey. We fit the $P K_{\mathrm{s}}$ relations of RR Lyrae stars in each tile, separately, and found a significant dispersion which is mostly owing to a depth effect.

To study the three-dimensional structure of the SMC we derived individual distances to $2997 \mathrm{RR}$ Lyrae stars by applying the near-infrared $P M_{K_{\mathrm{s}}} Z$ relation defined in Muraveva et al. (2015) to intensity-averaged $K_{\mathrm{s}}$ magnitudes from the VMC survey, periods from the OGLE IV catalogue and photometrically determined metallicities from Skowron et al. (2016) which we transformed to the metallicity scale defined by Gratton et al. (2004). The RR Lyrae variables in the SMC are found to have a roughly spheroidal 
or ellipsoidal distribution. We modelled the distribution of the SMC RR Lyrae stars as a triaxial ellipsoid and found the values of the axes ratio, position angle of the major axis of the ellipsoid projected on the sky and inclination of the longest axis with respect to the sky plane using inertia tensor analysis. The parameters of the SMC structure are: axes ratio $=1: 1.11( \pm 0.01): 3.30( \pm 0.70), 84^{\circ} .6 \pm 0^{\circ} .2$ and $2^{\circ} .1 \pm 0^{\circ} .6$. The results obtained in this paper are generally consistent with previous studies. Existing discrepancies are mostly owing to differences in the area of the SMC covered by the different studies.

The actual line-of-sight depth of the SMC has values in the range from 1 to $10 \mathrm{kpc}$, with an average depth of $4.3 \pm 1.0 \mathrm{kpc}$. The central parts of the SMC have larger depth. Taking into account the standard deviation associated with the mean distance modulus of our entire sample $(m-M)_{0}=$ $18.88 \pm 0.20$ mag we estimated a line-of-sight depth of the $\mathrm{SMC}$ as $5.2 \mathrm{kpc}$.

The spatial distribution of the RR Lyrae stars in our sample does not show features typical of young stellar populations, such as a bar. However, from the two-dimensional distribution of the extinction at different distances we can see the bar-like feature and the star forming region in the eastern Wing of the SMC. From our analysis of the SMC structure we concluded that the eastern part of the SMC is located closer to us than the western part. In eastern regions, beyond $2 \mathrm{deg}$ in radius from the centre, majority of the sub-regions have shorter distances. The regions where we see a large number of closer RR Lyrae stars are in the direction of SMC's trailing arm newly identified with Gaia (Belokurov et al. 2017). All these results indicate that the oldest stellar population in the eastern part of the SMC is affected by interactions of the Magellanic Clouds, occurred about 200-300 Myr ago which are believed to be mainly responsible for the formation of the MB.

Further study of RR Lyrae stars in the MB and outer regions of the LMC and SMC using near-infrared photometry will provide valuable information to understand the interaction history of the MS.

\section{ACKNOWLEDGEMENTS}

We thank the Cambridge Astronomy Survey Unit (CASU) and the Wide Field Astronomy Unit (WFAU) in Edinburgh for providing calibrated data products under the support of the Science and Technology Facility Council (STFC) in the UK. S.S acknowledges research funding support from Chinese Postdoctoral Science Foundation (grant number 2016M590013). M.-R.C. acknowledges support from STFC (grant number ST/M00108/1) and from the European Research Council (ERC) under the European Union's Horizon 2020 research and innovation programme (grant agreement No 682115). R. d. G. acknowledges financial support from the National Natural Science Foundation of China through grants 11373010, 11633005 and U1631102. 
Table 3. Properties of the 2997 RRab stars in the SMC analysed in the present paper: (1) VMC identification; (2) OGLE identification; (3) Number of the VMC tile; (4), (5) $V$ and $I$ magnitudes from the OGLE IV catalogue; (6) Pulsation period from OGLE IV; (7) Dereddened $K_{\mathrm{S}}$ magnitude; (8) Amplitude in $K_{\mathrm{S}}$; (9) Reddening; (10) Metallicity from Skowron et al. (2016) transformed to the metallicity scale defined by Gratton et al. (2004); (11) Distance modulus.

\begin{tabular}{|c|c|c|c|c|c|c|c|c|c|c|}
\hline VMC id & OGLE id & Tile & $\begin{array}{c}V \\
(\mathrm{mag})\end{array}$ & $\begin{array}{c}I \\
(\mathrm{mag})\end{array}$ & $\begin{array}{l}\text { Period } \\
\text { (days) }\end{array}$ & $\begin{array}{c}K_{\mathrm{s}, 0} \\
(\mathrm{mag})\end{array}$ & $\begin{array}{c}A m p\left(K_{\mathrm{s}}\right) \\
(\mathrm{mag})\end{array}$ & $\begin{array}{c}E(V-I) \\
(\mathrm{mag})\end{array}$ & $\begin{array}{c}{[\mathrm{Fe} / \mathrm{H}]} \\
(\mathrm{dex})\end{array}$ & $\begin{array}{c}(m-M)_{0} \\
\quad(\mathrm{mag})\end{array}$ \\
\hline J010326.45-712127.6 & OGLE-SMC-RRLYR-1768 & $6 \_4$ & 20.095 & 19.671 & 0.3742896 & $19.091 \pm 0.105$ & 0.212 & $0.03 \pm 0.05$ & $-1.790 \pm 0.330$ & $19.039 \pm 0.196$ \\
\hline J012415.27-721623.2 & OGLE-SMC-RRLYR-5285 & $5 \_5$ & 19.082 & 18.675 & 0.3939063 & $18.296 \pm 0.049$ & 0.230 & $-0.04 \pm 0.04$ & $-0.557 \pm 0.042$ & $18.268 \pm 0.119$ \\
\hline J004801.51-733021.8 & OGLE-SMC-RRLYR-0931 & $4 \_3$ & 19.813 & 19.502 & 0.3995731 & $18.820 \pm 0.052$ & 0.163 & $-0.10 \pm 0.04$ & $-1.790 \pm 0.330$ & $18.846 \pm 0.169$ \\
\hline J005300.26-725137.0 & OGLE-SMC-RRLYR-1218 & 4_3 & 19.965 & 19.554 & 0.4035819 & $19.132 \pm 0.077$ & 0.268 & $0.01 \pm 0.04$ & $-1.790 \pm 0.330$ & $19.170 \pm 0.178$ \\
\hline J003841.18-734423.1 & OGLE-SMC-RRLYR-0492 & 3_3 & 19.988 & 19.573 & 0.4105743 & $19.083 \pm 0.081$ & 0.170 & $-0.01 \pm 0.04$ & $-1.790 \pm 0.330$ & $19.142 \pm 0.178$ \\
\hline J003727.81-731455.3 & OGLE-SMC-RRLYR-0432 & $4 \_3$ & 19.949 & 19.509 & 0.4126961 & $19.113 \pm 0.069$ & 0.405 & $0.02 \pm 0.04$ & $-1.790 \pm 0.330$ & $19.178 \pm 0.173$ \\
\hline J005845.71-733438.3 & OGLE-SMC-RRLYR-1543 & $4 \_4$ & 20.245 & 19.768 & 0.4127270 & $19.335 \pm 0.112$ & 0.484 & $0.06 \pm 0.04$ & $-1.790 \pm 0.330$ & $19.400 \pm 0.194$ \\
\hline J005728.85-723454.9 & OGLE-SMC-RRLYR-1487 & $4 \_4$ & 20.317 & 19.794 & 0.4162569 & $19.328 \pm 0.103$ & 0.198 & $0.09 \pm 0.04$ & $-1.790 \pm 0.330$ & $19.403 \pm 0.188$ \\
\hline J010412.98-732541.8 & OGLE-SMC-RRLYR-1800 & $4 \_4$ & 20.158 & 19.614 & 0.4182084 & $19.118 \pm 0.109$ & 0.157 & $0.13 \pm 0.04$ & $-1.004 \pm 0.032$ & $19.174 \pm 0.161$ \\
\hline J005922.20-714625.5 & OGLE-SMC-RRLYR-1581 & 5_4 & 19.852 & 19.418 & 0.4210488 & $19.006 \pm 0.047$ & 0.491 & $0.01 \pm 0.04$ & $-1.118 \pm 0.029$ & $19.074 \pm 0.131$ \\
\hline J010215.39-741252.4 & OGLE-SMC-RRLYR-1697 & 3_4 & - & 19.580 & 0.423906 & $18.952 \pm 0.124$ & 0.136 & $0.06 \pm 0.06$ & $-1.790 \pm 0.330$ & $19.048 \pm 0.200$ \\
\hline J004639.17-731325.2 & OGLE-SMC-RRLYR-0862 & $4 \_3$ & 20.067 & 19.499 & 0.4243253 & $18.435 \pm 0.042$ & 22 & $0.15 \pm 0.04$ & $-1.790 \pm 0.330$ & $18.532 \pm 0.162$ \\
\hline J013735.79-744047.1 & OGLE-SMC-RRLYR-5749 & $2 \_5$ & 19.506 & 19.128 & 0.4264885 & $18.545 \pm 0.084$ & 0.321 & $-0.06 \pm 0.04$ & $-1.790 \pm 0.330$ & $18.648 \pm 0.177$ \\
\hline J005110.49-730750.4 & OGLE-SMC-RRLYR-1103 & $4 \_3$ & 20.036 & 19.611 & 0.4317203 & $19.493 \pm 0.090$ & 0.465 & $0.01 \pm 0.04$ & $-1.790 \pm 0.330$ & $19.611 \pm 0.180$ \\
\hline J010134.36-725427.7 & OGLE-SMC-RRLYR-1677 & 4_4 & 19.892 & 19.373 & 0.4335109 & $18.890 \pm 0.112$ & 0.200 & $0.06 \pm 0.04$ & $-1.790 \pm 0.330$ & $19.013 \pm 0.191$ \\
\hline J005719.65-725541.0 & OGLE-SMC-RRLYR-1476 & $4 \_4$ & 20.040 & 19.593 & 0.4337982 & $19.122 \pm 0.120$ & 0.316 & $0 \pm 0.04$ & $-1.790 \pm 0.330$ & $19.246 \pm 0.196$ \\
\hline J005126.22-715328.5 & OGLE-SMC-RRLYR-1117 & 5_3 & 19.981 & 19.496 & 0.4344378 & $18.990 \pm 0.094$ & 0.414 & $0.06 \pm 0.04$ & $-1.790 \pm 0.330$ & $19.116 \pm 0.181$ \\
\hline J003257.55-732906.3 & OGLE-SMC-RRLYR-0276 & $4 \_2$ & 20.169 & 19.688 & 0.4362651 & $19.100 \pm 0.126$ & 0.433 & $0.05 \pm 0.04$ & $-1.790 \pm 0.330$ & $19.230 \pm 0.199$ \\
\hline J003618.99-725748.0 & OGLE-SMC-RRLYR-0383 & 4_3 & 19.892 & 19.444 & 0.4373313 & $18.942 \pm 0.102$ & 0.461 & $0.01 \pm 0.04$ & $-1.790 \pm 0.330$ & $19.075 \pm 0.185$ \\
\hline J003229.44-731557.6 & OGLE-SMC-RRLYR-0262 & 4_2 & 19.604 & 19.316 & 0.4379744 & $19.081 \pm 0.093$ & 0.361 & $-0.18 \pm 0.04$ & $-0.992 \pm 0.048$ & $19.192 \pm 0.147$ \\
\hline J010814.34-735314.6 & OGLE-SMC-RRLYR-1975 & $3 \_4$ & 19.964 & 19.461 & 0.4420332 & $18.972 \pm 0.104$ & 0.139 & $0.07 \pm 0.04$ & $-1.790 \pm 0.330$ & $19.118 \pm 0.186$ \\
\hline J003418.32-724237.9 & OGLE-SMC-RRLYR-0321 & $4 \_2$ & 20.085 & 19.588 & 0.4451769 & $19.064 \pm 0.106$ & 0.380 & $0.06 \pm 0.04$ & $-1.790 \pm 0.330$ & $19.219 \pm 0.187$ \\
\hline J005646.18-723452.4 & OGLE-SMC-RRLYR-1440 & 5_4 & 20.031 & 19.516 & 0.4459867 & $19.105 \pm 0.159$ & 0.343 & $0.08 \pm 0.04$ & $-1.230 \pm 0.032$ & $19.245 \pm 0.202$ \\
\hline J010653.14-743637.7 & OGLE-SMC-RRLYR-4745 & 3_4 & 19.802 & 19.348 & 0.4484228 & $18.661 \pm 0.076$ & 0.386 & $0.03 \pm 0.04$ & $-1.199 \pm 0.032$ & $18.806 \pm 0.143$ \\
\hline J005509.35-735505.2 & OGLE-SMC-RRLYR-1358 & 3_3 & 19.870 & 19.399 & 0.4491077 & $18.949 \pm 0.065$ & 0.262 & $0.02 \pm 0.04$ & $-1.790 \pm 0.330$ & $19.114 \pm 0.166$ \\
\hline J005327.84-733656.3 & OGLE-SMC-RRLYR-1247 & 4_3 & 20.354 & 19.818 & 0.4497006 & $19.073 \pm 0.100$ & 0.332 & $0.09 \pm 0.04$ & $-1.790 \pm 0.330$ & $19.239 \pm 0.183$ \\
\hline J005629.88-710719.3 & OGLE-SMC-RRLYR-4342 & $6 \_4$ & 19.879 & 19.393 & 0.4497817 & $18.901 \pm 0.120$ & 0.588 & $0.03 \pm 0.04$ & $-1.790 \pm 0.330$ & $19.068 \pm 0.194$ \\
\hline J004758.96-732241.7 & OGLE-SMC-RRLYR-0928 & $4 \_3$ & 20.400 & 19.898 & 0.4497919 & $19.341 \pm 0.163$ & 0.162 & $0.07 \pm 0.04$ & $-1.790 \pm 0.330$ & $19.508 \pm 0.224$ \\
\hline J010535.93-720621.9 & OGLE-SMC-RRLYR-1867 & $5 \_4$ & 19.932 & 19.503 & 0.4538355 & $18.873 \pm 0.087$ & 0.278 & $-0.02 \pm 0.04$ & $-1.790 \pm 0.330$ & $19.050 \pm 0.175$ \\
\hline J003057.78-735500.2 & OGLE-SMC-RRLYR-0216 & 3_2 & 19.765 & 19.379 & 0.4545178 & $18.910 \pm 0.059$ & 0.418 & $-0.07 \pm 0.04$ & $-1.241 \pm 0.041$ & $19.072 \pm 0.136$ \\
\hline J010516.58-722526.8 & OGLE-SMC-RRLYR-1854 & $5 \_4$ & 20.087 & 19.525 & 0.4559563 & $19.105 \pm 0.044$ & 0.276 & $0.12 \pm 0.04$ & $-1.083 \pm 0.033$ & $19.266 \pm 0.123$ \\
\hline J003651.40-713108.5 & OGLE-SMC-RRLYR-3606 & 5_3 & 19.913 & 19.455 & 0.4563110 & $18.859 \pm 0.138$ & 0.182 & $0.01 \pm 0.04$ & $-1.790 \pm 0.330$ & $19.043 \pm 0.205$ \\
\hline J011942.83-742418.6 & OGLE-SMC-RRLYR-5163 & $3 \_5$ & 19.771 & 19.224 & 0.4576531 & $18.422 \pm 0.080$ & 0.206 & $0.11 \pm 0.04$ & $-1.217 \pm 0.029$ & $18.592 \pm 0.145$ \\
\hline
\end{tabular}

The table is published in its entirety in the electronic version of the paper. A portion is shown here for guidance regarding its format and content. 


\section{REFERENCES}

Belokurov V., Erkal D., Deason A. J., Koposov S. E., De Angeli F., Evans D. W., Fraternali F., Mackey D., 2017, MNRAS, 466,4711

Besla G., Kallivayalil N., Hernquist L., van der Marel R. P., Cox T. J., Kereš, D., 2012, MNRAS, 421, 2109

Bono G., Caputo F., Castellani V., Marconi M., Storm J., Degl'Innocenti S., 2003, MNRAS, 344, 1097

Borissova J., Rejkuba M., Minniti D., Catelan M., Ivanov V. D., 2009, A\&A, 502, 505

Caputo F., Marconi M., Musella I., 2000, A\&A, 354, 610

Cardelli J. A., Clayton G. C., Mathis J. S., 1989, ApJ, 345, 245

Catelan M., Pritzl B. J., Smith H. A. E., 2004, ApJS, 154, 633

Cioni M.-R. L. et al., 2011, A\&A, 527, 116

Clementini G. et al., 2000, AJ, 120, 2054

Clementini G., Gratton R., Bragaglia A., Carretta E., Di Fabrizio L., Maio M. et al., 2003, AJ, 125, 1309

Clementini G. et al. 2016, A\&A, 595, 133

Cross N. J. G. et al., 2012, A\&A, 548, A119

de Grijs R., Bono G., 2015, AJ, 149, 179

De Propris R., Rich R. M., Mallery R. C., Howard C. D., 2010, ApJ, 714, L249

Deb S., Singh H. P., Kumar S., Kanbur S. M., 2015, MNRAS, 449,2768

Deb S., 2017, preprint (arXiv: 1707.03130)

Del Principe M. et al., 2006, ApJ, 652, 362

Diaz J. D., Bekki K., 2012, ApJ, 750, 36

Di Criscienzo M., Marconi M., Caputo F., 2004, ApJ, 612, 1092

Gaia Collaboration et al. 2016a, A\&A, 595, A2

Gaia Collaboration et al. 2016b, A\&A, 595, A1

Graham J. A., 1975, PASP, 87, 641

Gratton R. G., Bragaglia A., Clementini G., Carretta E., Di Fabrizio L., Maio M., Taribello E., 2004, A\&A, 421, 937

Haschke R., Grebel E. K., Duffau S., 2011, AJ, 141, 158

Haschke R., Grebel E. K., Duffau S., 2012, AJ, 144, 107

Israel F. P., Wall W. F., Raban D., Reach W. T., Bot C., Oonk J. B. R., Ysard N., Bernard J. P., 2010, A\&A, 519, A67

Jacyszyn-Dobrzeniecka A. M. et al., 2017, Acta Astron., 67, 1

Jarvis M. J. et al., 2013, MNRAS, 428, 1281

Jurcsik J., 1995, Acta Astron., 45, 653.

Kapakos E., Hatzidimitriou D., 2012, MNRAS, 426, 2063.

Lewis J. R., Irwin M., Bunclark P., 2010, in Yoshihiko M., KohIchirp M., Masatoshi O., eds, ASP Conf. Ser. Vol. 434, Astronomical Data Analysis Software and Systems XIX. Astron. Soc. Pac., San Francisco, p. 91

Longmore, A. J., Fernley, J. A., Jameson, R. F., 1986, MNRAS, 220,279

Marconi M. et al., 2015, ApJ, 808, 50

Marconi M. et al., 2017, MNRAS, 466, 3206

Moretti M. I. et al., 2014, MNRAS437, 2702

Moretti M. I. et al., 2016, MNRAS, 459, 1687

Muraveva T. et al., 2014, MNRAS, 443, 432

Muraveva T. et al., 2015, ApJ, 807, 127

Nidever D. L., Majewski S. R. Muñoz R. R., Beaton R. L., Patterson R. J., Kunkel W. E., 2011, ApJ, 733, 10

Nidever D. L., Monachesi A., Bell E. F., Majewski S. R., Muñoz R. R., Beaton R. L., 2013, ApJ, 779, 145

Paz D. J., Lambas D. G., Padilla N., Merchán M., 2006, MNRAS, 366,1503

Pejcha O., Stanek K. Z., 2009, ApJ, 704, 1730

Piersimoni A. M., Bono G., Ripepi V. 2002, AJ, 124, 1528

Putman et al., 1998, Nature, 394, 752

Ripepi V. et al., 2012a, MNRAS, 424, 1807

Ripepi V., Moretti M. I., Clementini G., Marconi M., Cioni M. R., Marquette J. B., Tisserand P., 2012b, Ap\&SS, 341, 51

Ripepi V. et al., 2014, MNRAS, 437, 2307

Ripepi V. et al., 2015, MNRAS, 446, 3034
Ripepi V. et al., 2016, ApJS, 224, 21

Ripepi V. et al., 2017, submitted to MNRAS

Rubele S. et al., 2015, MNRAS, 449, 639

Schlegel D. J., Finkbeiner D. P., Davis M., 1998, ApJ, 500, 525

Skowron D. M. et al., 2016, Acta Astron., 66, 269

Sollima, A., Cacciari, C., Valenti, E., 2006, MNRAS, 372, 1675

Sollima A., Cacciari C., Arkharov A. A. H., Larionov V. M., Gorshanov D. L., Efimova N. V. , Piersimoni A., 2008, MNRAS, 384,1583

Soszyński I., Udalski A., Szymański M. K., Kubiak J., Pietrzyński G., Wyrzykowski Ł., Ulaczyk K., Poleski R., 2010, Acta Astron., 60,165

Soszyński I. et al., 2016, Acta Astron., 66, 131

Stanimirović S., Staveley-Smith L., Jones P. A., 2004, ApJ, 604, 176

Subramanian S., Subramaniam A., 2012, ApJ, 744, 128

Subramanian S. et al., 2017, MNRAS, 467, 2980

Tatton B. L. et al., 2013, A\&A, 554, A33

Udalski A., Szymański M. K., Szymański G., 2015, Acta Astron., 65,1

van der Marel R. P., Cioni M.-R. L., 2001, AJ, 122, 1807

Walker A. R., Mack P., 1988, AJ, 96, 872

Zaritsky D., Harris J., Thompson I. B., Grebel E. K., Massey P., 2002, AJ, 123, 855

Zinn R., West M. J., 1984, ApJS, 55, 45

This paper has been typeset from a $\mathrm{T}_{\mathrm{E}} \mathrm{X} / \mathrm{L}_{\mathrm{A}} \mathrm{X}$ file prepared by the author. 